\title{
TRANSIENT MEMBRANE SHEDDING IN LIMULUS PHOTORECEPTORS: CONTROL MECHANISMS UNDER NATURAL LIGHTING ${ }^{1}$
}

\author{
STEVEN C. CHAMBERLAIN ${ }^{2}$ AND ROBERT B. BARLOW, JR. \\ Institute for Sensory Research, Syracuse University, Syracuse, New York 13210
}

Received February 8, 1984; Revised May 25, 1984; Accepted May 30, 1984

\begin{abstract}
Photoreceptors of the Limulus lateral eye shed their light-sensitive membranes (rhabdoms) in a burst early each morning when the animal is maintained in natural lighting. This shedding burst produces a cloud of multivesicular bodies which coalesce and migrate away from the rhabdom. Within $24 \mathrm{hr}$, these gradually collapse to combination bodies and ultimately to lamellar bodies.

Light initiates the burst of shedding. If animals are maintained in darkness beyond their normal dawn, the shedding burst is delayed until the first onset of light. We have not been able to produce a second burst of membrane shedding within one 24 -hr period.

Efferent optic nerve activity generated by a circadian clock in the brain primes the shedding burst. At least $3 \mathrm{hr}$ of efferent activity in darkness must precede light onset to prime membrane shedding; however, the efferent fibers need not be active when the light-initiated burst occurs. Chronically blocking the efferent input to the retina abolishes the shedding burst.

The burst of membrane shedding is robust and short-lived. Within $15 \mathrm{~min}$ of light onset the area of photosensitive membrane decreases by about $70 \%$, and within an hour the rhabdom returns to essentially its preburst size. At other times in the diurnal light cycle, the size of the rhabdom undergoes significant variations which are not abolished by blocking the efferent input. Apparently the daily burst of shedding overlays a second cycle of membrane metabolism that is not controlled by efferent optic nerve activity.
\end{abstract}

Photoreceptors catch light and convert it to the electrical signals which lead ultimately to seeing. To do this, they have developed specialized organelles, such as the stacked disks of vertebrate rods and cones and the microvillar arrays of invertebrate retinular cells. The photosensitive membrane of these organelles appears to be unstable in some intrinsic way since it is broken down, or shed, and synthesized on a regular basis. Normally, the processes of breakdown and renewal are in precise balance, because the total amount of photosensitive membrane in each cell may remain essentially constant over periods of years. Such balance between opposing cellular processes implies elegant control mechanisms which must operate successfully over long periods of time and under varied environmental conditions. In this paper we report some important

\footnotetext{
${ }^{1}$ This research was supported by National Institutes of Health Grants EY-03446 and EY-00667, National Science Foundation Grant BNS 82-41893, and the Grass Foundation. We thank William Dossert, Robert Fladd, Leslie Eisele, and Joseph Fladd for technical assistance. We thank Barabara-Anne Battelle, W. Henner Fahrenbach, Alan Fein, Len Kass, Larry A. Westerman, Richard H. White, and David S. Williams for helpful discussions during the progress of this work. We thank the Department of Anatomy, State University of New York Upstate Medical Center for the use of their electron microscope and computer facilities for portions of this research.

${ }^{2}$ To whom correspondence should be addressed.
}

aspects of the way in which the shedding of photoreceptor membrane is controlled in the lateral eye of the horseshoe crab, Limulus polyphemus.

The visual system of Limulus has proven useful for the study of basic mechanisms of retinal function (e.g., Ratliff, 1974; Fain and Lisman, 1981; Fein and Szuts, 1982). Recent studies of the lateral eye have expanded this body of knowledge to include information about the central control of peripheral visual function. Efferent fibers from the brain innervate the retina of the lateral eye and make direct contact with the photoreceptors (Fahrenbach, 1973, 1981; Barlow and Chamberlain, 1980). These fibers are connected to a central circadian clock and transmit nerve impulses to the eye at night (Barlow et al., 1977; Barlow, 1983). Efferent activity modulates the structure of retinal cells (Chamberlain and Barlow, 1977; Barlow and Chamberlain, 1980), increases the acceptance angle of individual ommatidia (Barlow et al., 1980b), decreases photoreceptor noise (Kaplan and Barlow, 1980), decreases retinal inhibitory interactions (Batra and Barlow, 1982), increases retinal gain (Barlow and Chamberlain, 1980), and enables photomechanical movements (Chamberlain and Barlow, 1981).

Here we report that efferent activity and diurnal lighting play important roles in controlling the daily shedding of photosensitive membrane in the lateral eye. We show that a daily rapid burst of shedding is jointly controlled by efferent input from the circadian clock at night and by light onset at dawn. We have studied the shedding process under conditions of both 
natural and artificial lighting. A preliminary report of portions of this work has appeared elsewhere (Chamberlain and Barlow, 1979).

\section{Materials and Methods}

Fixation and embedding. Male and female adult horseshoe crabs (Limulus polyphemus) measuring 20 to $25 \mathrm{~cm}$ across the carapace were used for this study. For experiments carried out in June at the Marine Biological Laboratory (Woods Hole, MA), freshly collected animals were stored under natural lighting in aquaria with circulated seawater or in pens in the ocean. For experiments performed during other months in our laboratories in Syracuse, animals were maintained under fluorescent lighting in aquaria with recirculated artificial seawater (Instant Ocean, Aquarium Systems, Inc., Eastlake, $\mathrm{OH}$ ) and were regularly fed fresh clams. The light/dark cycle matched that of local natural lighting. Except in control experiments, all retinas were fixed without change in ambient illumination by subretinal injection of a fixative containing $4.5 \%$ sucrose, $3 \% \mathrm{NaCl}, 0.8 \%$ glutaraldehyde, and $5 \%$ formaldehyde in $0.1 \mathrm{M}$ Sorensen's phosphate buffer at $\mathrm{pH} 7.2$ (Fahrenbach, 1969). After remaining $30 \mathrm{~min}$ in the appropriate condition of light or dark, the retina and cornea were then dissected free and fixed overnight by immersion in fixative at $4^{\circ} \mathrm{C}$. They were then washed in $8 \%$ sucrose in phosphate buffer, cut into small blocks, and postfixed in $1 \% \mathrm{OsO}_{4}$ in phosphate buffer for $1 \mathrm{hr}$ at room temperature. Finally they were washed in distilled water, dehydrated through an ethanol series to propylene oxide, and embedded in Epon-Araldite.

In control experiments to determine whether the formation of membrane whorls was caused by the method of fixation, one eye was fixed as described above and the other was fixed by one of the following four alternative procedures. (1) The retina was dissected free and fixed by immersion in $40 \% \mathrm{OsO}_{4}$ in $\mathrm{CCl}_{4}$ (Afzelius, 1959; Krebs and Schaten, 1976) for $20 \mathrm{~min}$ at room temperature, dehydrated, and embedded. (2) The retina was fixed by subretinal injection of a fixative containing $4.5 \%$ sucrose, $3 \% \mathrm{NaCl}, 5 \mathrm{mM} \mathrm{CaCl}, 0.8 \%$ glutaraldehyde, and $5 \%$ formaldehyde in $0.08 \mathrm{M}$ cacodylate buffer at $\mathrm{pH} 7.4$, dissected free immersed in fixative overnight at $4{ }^{\circ} \mathrm{C}$, washed in $8 \%$ sucrose in $0.1 \mathrm{M}$ cacodylate buffer, postfixed in $1 \% \mathrm{OsO}_{4}$ for $1 \mathrm{hr}$, and dehydrated and embedded as above. (3) The retina was fixed by subretinal injection of the standard fixative to which $2 \mathrm{~mm}$ EGTA was added (Williams, 1980 ), dissected free, fixed by immersion in fixative overnight at $4^{\circ} \mathrm{C}$ and processed by the standard methods above. (4) The retina was fixed by the normal method, but only for $1 \mathrm{hr}$, and then processed by the standard methods above.

Light microscopy. For observation with the light microscope, serial $1-\mu \mathrm{m}$ sections were cut with a Sorvall MT2-B ultramicrotome (DupontSorvall, Newtown, CT) using glass knives. Each plastic block contained about 40 ommatidia. Sections were oriented to be perpendicular to the optic axes of ommatidia in the center of each block. Sections were mounled on glass slides and stained with $1 \%$ toluidine blue (Trump et al., 1961). The locations of sections on slides were mapped, and then the extent of the rhabdom in the serial set was determined by finding the section with the top of the rhabdom just under the cuticular cone and the section with the bottom of the rhabdom near the eccentric cell soma. The section midway between these extremes was photographed with a Nikon Biophot microscope (Nikon, Inc., Garden City, NY), and prints were prepared for planimetry.

Electron microscopy. Gold and silver thin sections were cut with glass knives on either a Sorvall MT2-B or MT5000 ultramicrotome and placed on naked copper grids. Sections were stained with uranyl acetate and lead citrate. Electron micrographs were prepared using a Siemens Elmiskop 101 electron microscope (Siemens Corp., Iselin, NJ). Most sections were cut perpendicular to the optic axes of the ommatidia. For examination of the microvillar array in cross-section, sections were cut parallel to the ommatidial axis.

Computer-assisted planimetry. As described in detail under "Results," the area of rhabdomeral membrane was determined from two types of planimetric measurements. To determine the average length of microvilli for each condition, light micrographs were used. The center serial section of each of 20 ommatidia was photographed and printed. The four rays with the smallest thickness were chosen from each rhabdom array to minimize the effects of distortion caused by minor departures from the ideal plane of section. These four rays were either adjacent or opposite pairs. The area of each ray was determined by digitizing its perimeter with an acoustic tablet and determining the enclosed area with a planimetry program. Then the center line was digitized, and the area was divided by the center line length to determine twice the average microvillar length. (Each ray of the rhabdom is made up of two layers of abutting microvilli.) Thus $80(4 \times 20)$ samples were averaged to determine the mean microvillar length for each condition. To determine the cross-sectional dimensions and packing density of microvilli in the rhabdom, we selected electron micrographs from the appropriate sections. The perimeters of cross-sections of 100 microvilli were digitized for each condition, and the perimeter length and enclosed area were determined. The mean area measurements were then used to calculate the packing density of microvilli in the rhabdom, and the mean perimeter lengths were used to calculate the membrane area per microvillus.

Electrophysiology. In some experiments, one or both lateral optic nerves were cut in an otherwise intact animal, and the peripheral portion of one nerve was electrically stimulated to mimic efferent activity while the electroretinogram (ERG) of the innervated eye was monitored. The electrophysiological methods are described in detail elsewhere (Barlow, 1983). Briefly, the animal was clamped to a rigid platform in an aquarium located in a lightproof, shielded cage. The aquarium was filled with artificial seawater to the level of the lateral eyes, and the gill structure was continuously washed with aerated seawater. The lateral eye ERG in response to a brief flash was recorded with a corneal electrode. Current pulses were delivered to the peripheral stump of the cut lateral optic nerve via a suction electrode and pulse stimulator (models PS1U6 and S44, Grass Instrument Co., Quincy, MA). The stimulation pattern of 2 pulses/sec for 30 sec every minute approximated that of endogenous efferent activity recorded at night (Barlow et al., 1977).

\section{Results}

\section{Summary anatomy of the Limulus lateral eye}

The basic microanatomy of the photoreceptors of the lateral eye has been described before (Miller, 1957, 1958; Lasansky, 1967; Fahrenbach, 1968, 1969, 1973, 1975, 1981; Cohen, 1973; Barlow and Chamberlain, 1980). Here, however, we have cast the microanatomy in the $\mathrm{R}$ - and A-segment dichotomy based on the division of ventral photoreceptors into $\mathrm{R}$ - and A-lobes (Calman and Chamberlain, 1982). We favor this terminology because it simplifies the description and makes clear the analogy to inner and outer segments of vertebrate photoreceptors.

Figure 1 shows diagrams of the position of the lateral eye, the arrangement of ommatidia within the eye, the structure of the ommatidia, and the segmentation of the photoreceptors. The $\mathrm{R}$-segment bears the rhabdomere; each ray of the rhabdom is formed by the abutting microvilli of two adjacent $R$-segments. The A-segment contains the nucleus and major metabolic machinery of the neuron. Efferent fibers originating in the central nervous system innervate all cell types of the ommatidium (Fahrenbach, 1973, 1981; Barlow and Chamberlain, 1980) but are especially numerous at the junction between the two segments of the photoreceptors.

\section{Daily rhythm in lateral eye structure}

Circadian and diurnal changes in the Limulus lateral eye form an important substrate for our presentation of the daily cycle of photoreceptor membrane shedding. In continuous darkness, efferent activity causes circadian rhythms in retinal structure (Behrens, 1974; Chamberlain and Barlow, 1977; Barlow et al., 1980). In natural lighting, the amplitudes of the cyclic changes in retinal structure are increased (Chamberlain and Barlow, 1981). At night, when the eye is well adapted to darkness, the aperture between the lens and the rhabdom opens wide to admit the greatest amount of light, and the rhabdom closely apposes the tip of the lens, folding to concentrate the photosensitive membrane just below the aperture. During the day, when the eye is well adapted to light, the aperture lengthens and narrows, and the rhabdom elongates and withdraws from the tip of the lens. The eye is in one of these two 


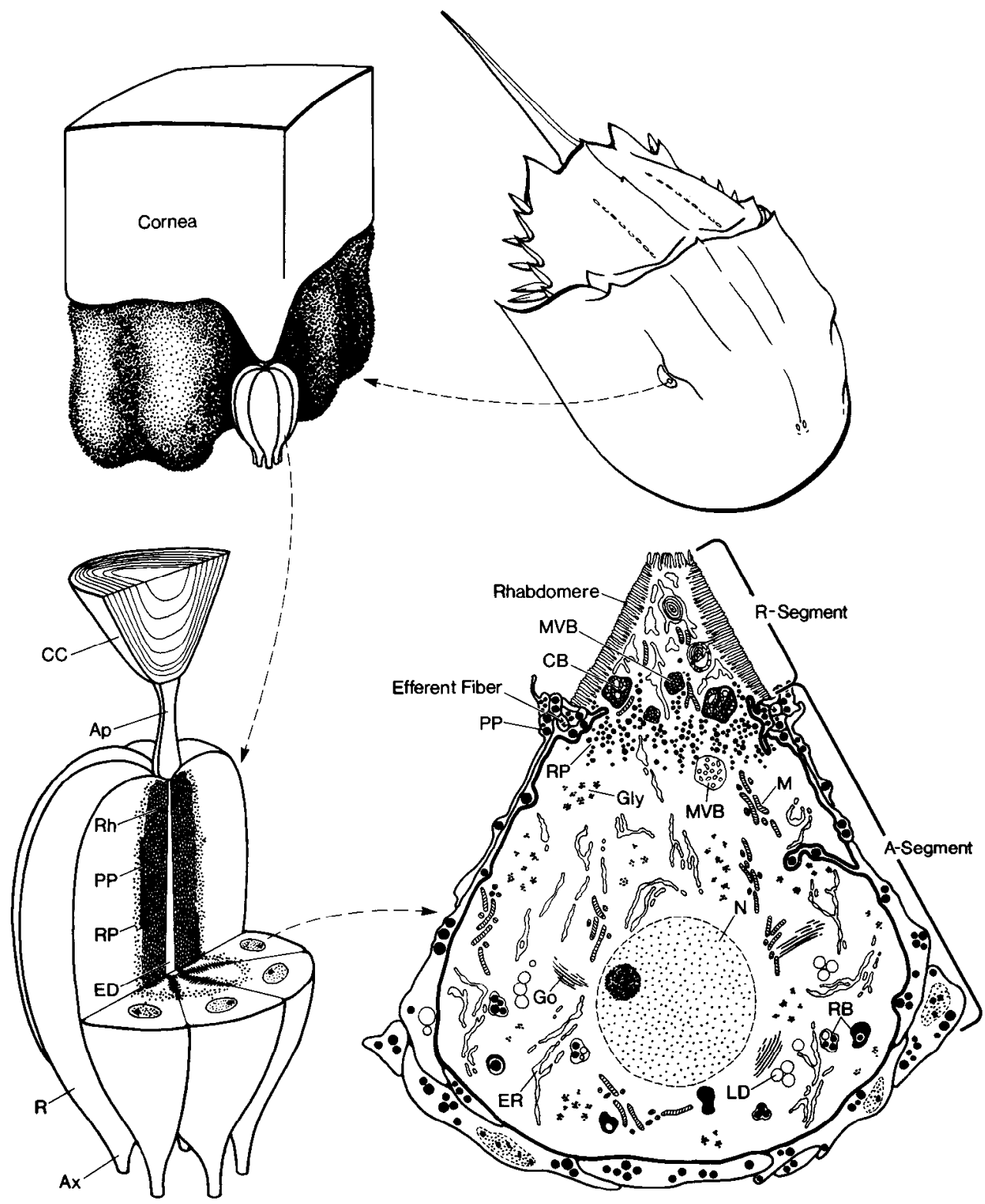

Figure 1. Summary anatomy of the light-adapted, daytime Limulus lateral eye. The sketch at the upper right shows the position of the lateral eye in the adult animal. At upper left is a small block of cornea and retina excised from the lateral eye as indicated. The ensheathing pigment cells have been stripped away from one ommatidium. The underlying neural plexus is not shown. At lower left is a cut-away view of a single ommatidium with structure typical of a daytime, light-adapted eye. The array of retinular cells $(R)$ is connected to the base of the cuticular cone $(C C)$ by a long narrow aperture $(A p)$. The rays of the rhabdom $(R h)$ surround the dendrite of the second-order eccentric cell $(E D)$. Pigment granules in the retinular cells $(R P)$ form a circular band around the peripheral ends of the rhabdom rays. Pigment granules in pigment cells $(P P)$ form columns at the end of each ray. Fewer than the usual 12 retinular cells are illustrated for clarity. At lower right is a retinular cell cut perpendicular to the optic axis of the ommatidium and labeled to define the rhabdomeral $(R)$-segment and the arhabdomeral $(A)$-segment. The surface of the $\mathrm{R}$-segment is covered by the microvillar array of the rhabdomere. The cytoplasm contains endoplasmic reticulum, mitochondria, and structures such as multivesicular bodies $(M V B)$ and combination bodies $(C B)$ which are part of the degradation pathway for shed microvillar membrane. During the day, retinular pigment granules $(R P)$ are found at the junction of the $\mathrm{R}$ - and $\mathrm{A}$-segments. The surface of the A-segment is covered hy pigment cells $(P P)$. Efferent fibers from the brain are found among the cluster of pigment cell profiles at the junction between the two segments. The A-segment contains the nucleus $(N)$, glycogen rosettes $(G l y)$, Golgi apparatus $(G o)$, lipid droplets $(L D)$, residual bodies $(R B)$, mitochondria $(M)$, endoplasmic reticulum $(E R)$, multivesicular bodies, and generally those structures found in neural somata. The structures in the sketch at lower right are not all drawn to scale.

states during most of the 24 -hr period with relatively brief transitions in the early morning and evening.

When observed with the light microscope, the rhabdom is prominent and well organized throughout the 24-hr cycle, ex- cept for a brief period in early morning when the rhabdom is broken down and rebuilt (Fig. 2). $\Lambda$ t the ultrastructural level the microvilli of the rhabdom are most extensive and best organized late at night, when they are well adapted to darkness 

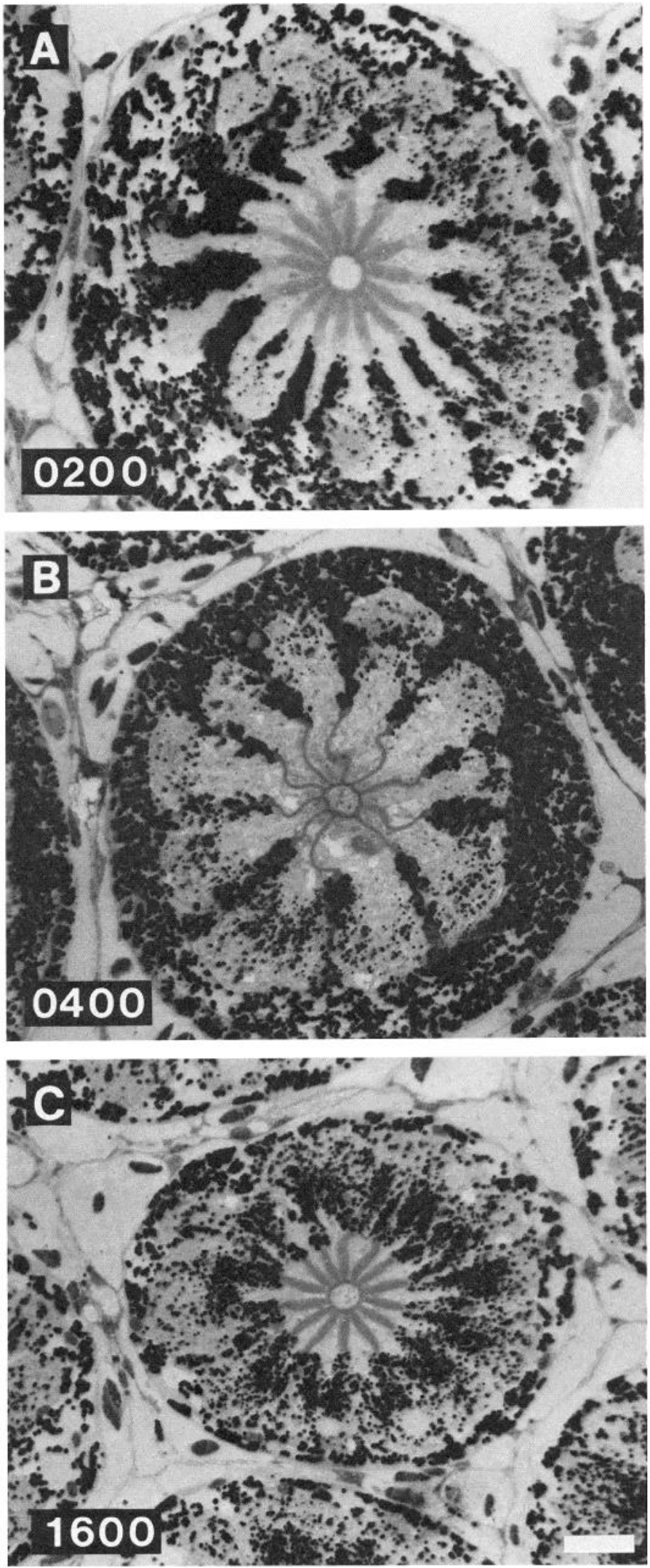

Figure 2. Light micrographs of Limulus ommatidia fixed in natural lighting. The $1-\mu \mathrm{m}$ plastic sections shown are all from the center of a serial set cut perpendicular to the optic axis of the ommatidium. $A$ shows the typical dark-adapted, nighttime structure. The overall crosssectional area of the ommatidium is large. The massed granules of pigment cells at the periphery of the rhabdom rays are prominent. The retinular cell pigment is dispersed to the periphery of the A-segment.
(Fig. $3 A$ ), and late in the afternoon, when they are well adapted to light (Fig. 6A).

\section{Daily cycle of photoreceptor membrane shedding in natural lighting}

Photoreceptors shed their light-sensitive membrane in a burst very early in the morning while the overall retinal structure is still in the dark-adapted nighttime state or is just beginning the transition to the light-adapted daytime state. During the shedding burst, the rhabdom is transiently disorganized. Subsequently, shed membrane forms cytoplasmic organelles which migrate from a position immediately adjacent to the rhabdomere, across the rhabdomeral segment, and into the arhabdomeral segment during the 24 -hr period subsequent to the shedding burst.

Predawn structure: $0200 \mathrm{hr}$. Late at night $(0200 \mathrm{hr})$, the microvilli of the rhabdom are long and thin and are well organized (Fig. 3A). The microvilli form quintuple-layered membranes along most of their contacts with each other (Fig. $3 B$ ). The cytoplasm of the rhabdomeral segment (R-segment) consists mainly of the subrhabdomeral palisades with occasional membrane-bound inclusions of pallid vesicles. In the periphery of the arhabdomeral segment (A-segment) residual bodies are commonly found which show alternate lucent areas with flocculent material and concentric rings of lamellae (Fig. $3 C$ ). These residual bodies turn out to be the final stages in the breakdown of membrane shed the previous morning.

Burst of membrane shedding: 0400 to $0600 \mathrm{hr}$. In late June the daily burst of photoreceptor membrane shedding occurs between 0400 and $0600 \mathrm{hr}$, local time. The microvilli of the rhabdom undergo a transient structural instability, forming membrane whorls which balloon into the cytoplasm of the Rsegment (Fig. 3, $D$ and $F$ ). During this period the quintuplelayered junctions between microvilli disappear over large distances of apposition (Fig. $3 E$ ). The membrane whorls are composed of up to seven concentric layers of microvillar membrane. The center spaces resemble either microvillar core material or subrhabdomeral palisades, although occasionally a mitochondrion is trapped within a membrane whorl. Clearly the formation of whorls requires the breaking and re-forming of continuous membranes. We have not observed any coated vesicles connected to the membrane whorls. The extent of shedding is much less for the microvilli of the central ring of the rhabdom array. Thus the single layer of interdigitated microvilli between the eccentric cell dendrite and the retinular cells is largely spared the degree of disorganization which characterizes the breakdown of the rhabdom between retinular cells. During membrane whorl formation, the cytoplasm of the eccentric cell dendrite is more osmophilic and is more darkly stained by toluidine blue than at any other time of the day.

The timing of the shedding burst varies across a population of animals and between the eyes of one animal. Thus, the timing of the evolution of breakdown organelles in the remainder of the daily cycle will have an uncertainty which we estimate does not exceed $2 \mathrm{hr}$ for the animals in the present study.

By $0600 \mathrm{hr}$, up to $2 \mathrm{hr}$ after the formation of membrane

The rays of the rhabdom are prominently developed. $B$ shows the structure during transient shedding of microvillar membrane. The overall structure is much the same as in $A$, except that the rays of the rhabdom are attenuated, and whorls of membrane are just visible in the cytoplasm of the R-segment between the rays of rhabdom. $C$ shows the typical light-adapted, daytime structure. The overall cross-sectional area is reduced because the ommatidium is elongated. The retinular pigment is aggregated to form a ring around the rhabdom, but most of it remains in the A-segment with relatively little appearing in the $\mathrm{R}$ segment between the rays of rhabdom. As in $A$, the rays of the rhabdom are prominently developed. The numbers at the lower left give the local time of fixation on a clear day in late June. The bar represents $26 \mu \mathrm{m}$. 

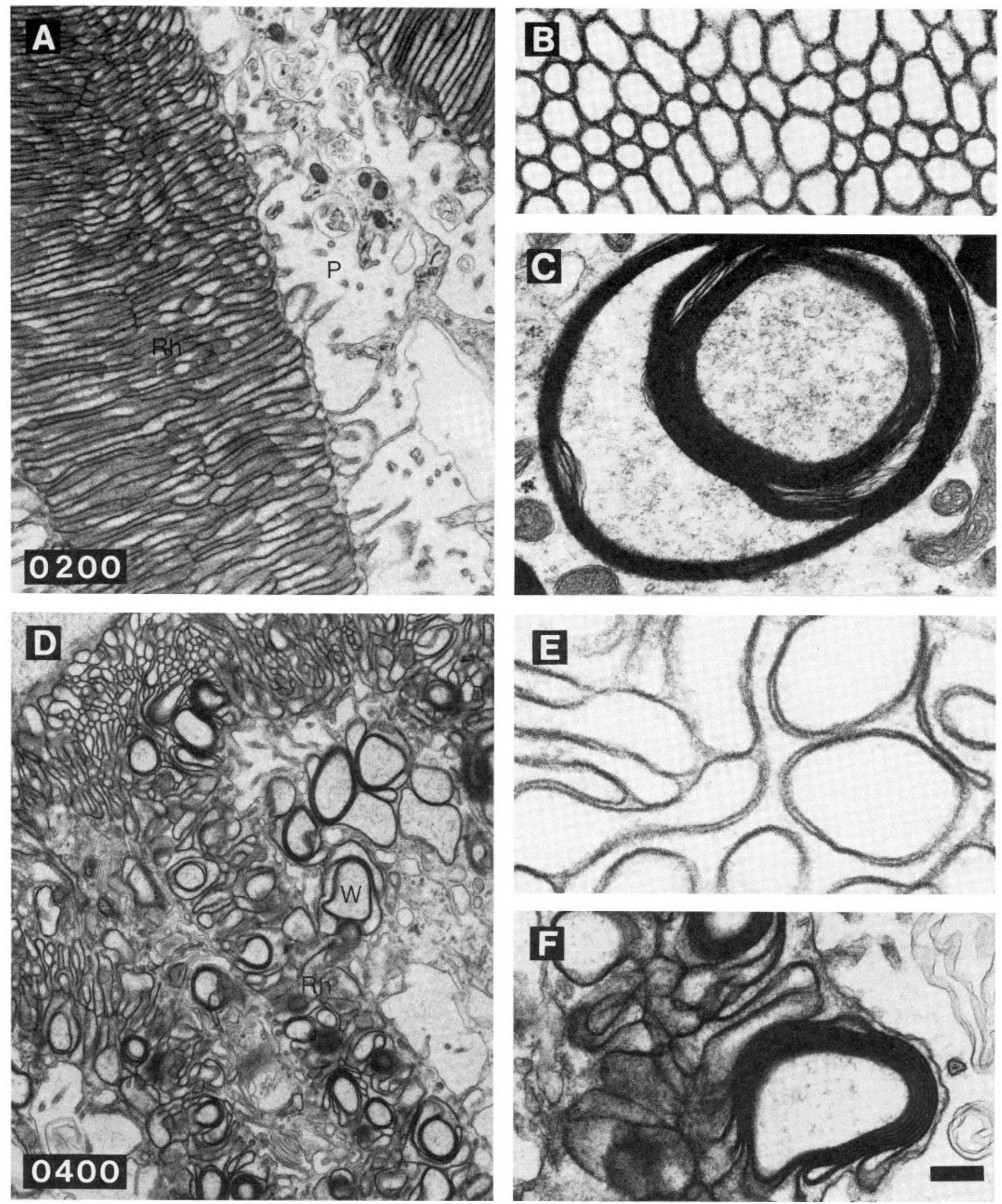

Figure 3. Ultrastructure of eyes fixed in early morning. $A$ to $C$, At $0200 \mathrm{hr}$ the rhabdom $(R h)$ is prominently developed and well organized.

The majority of the R-segment cytoplasm is occuppied by subrhabdomeral palisades $(P)$. The plane of section in $A$ is the same as for Figure 2 . $B$, The rhabdom has been cut perpendicular to the microvillar axes. The microvilli have relatively small diameters and their membranes are largely fused with those of adjacent microvilli at points of contact. $C$, Lamellar bodies remain in the A-segment from the transient shedding of microvillar membrane the preceding morning. $D$ to $F$, At $0400 \mathrm{hr}$ transient shedding of microvillar membrane is characterized by the formation of whorls of membrane $(W)$ in and adjacent to the rhabdom $(R h)$. $E$, When viewed in cross-section, the microvillar diameters are enlarged compared to $B$, and the total amount of photosensitive membrane is reduced. The regions of fusion of adjacent microvillar membranes also appear to be reduced. $F$, Membrane whorls are formed from loops of microvillar membrane. The $b a r$ in $F$ represents $0.56 \mu \mathrm{m}$ in $A$ and $D, 0.1 \mu \mathrm{m}$ in $B$ and $E$, and $0.24 \mu \mathrm{m}$ in $C$ and $F$. 
whorls, the rhabdom is again relatively well organized (Fig. $4 A$ ). Quintuple-layered junctions have been restored between the microvilli (Fig. $4 B$ ). The cytoplasm of the R-segment immediately adjacent to the rhabdom is filled with small, tightly assembled multivesicular bodies and clouds of single vesicles which appear to be coalescing into multivesicular bodies (Fig. $4 C$ ). Note in Figure $4 A$ (arrowheads) the presence of a few membrane whorls which have the same appearance as those in Figure $3 D$ except that the concentric quintuple-layered membranes have split apart and appear to be folding up. Although these structures may represent an intermediate stage between membrane whorls and multivesicular bodies, they may also represent something quite different, since these "split-apart" whorls persist during most of the day.

Daylight period: 0800 to $1800 \mathrm{hr}$. At $0800 \mathrm{hr}$ (Fig. $4 \mathrm{D}$ ) and $1000 \mathrm{hr}$ (Fig. 4E) the tightly assembled multivesicular bodies have the same appearance as earlier, except that they become progressively larger and move away from the rhabdom and toward the A-segment. By $1000 \mathrm{hr}$ a small percentage of the multivesicular bodies has begun to collapse into combination bodies with vesicular and lamellar zones. At $1200 \mathrm{hr}$ the majority of the multivesicular bodies are now at the junction between the R-segment and the A-segment and have begun collapsing into combination bodies (Fig. 5C). At $1400 \mathrm{hr}$ (Fig. 5D) about half of the multivesicular bodies have collapsed into combination bodies, and by $1600 \mathrm{hr}$ (Fig. $5 E$ ) the majority of the multivesicular bodies have collapsed. By $1800 \mathrm{hr}$, most of the combination bodies have converted to lamellar bodies which are interspersed among the retinular pigment granules in the A-segment (Fig. 6C).

During the hours of daylight, the microvilli of the rhabdom are well organized, with no remaining indication of membrane whorl formation such as that seen at $0600 \mathrm{hr}$. At $1200 \mathrm{hr}$ (Fig. 5, $A$ and $B$ ) and $1800 \mathrm{hr}$ (Fig. 6, $A$ and $B$ ), the well lightadapted rhabdom resembles the well dark-adapted rhabdom of night (cf. Figs. $3 A$ and $6 A$ ). However, at $1800 \mathrm{hr}$, the rhabdom does not have the prominent subrhabdomeral palisade present at $0200 \mathrm{hr}$, and there appears to be significantly more extracellular space between the microvilli at $1800 \mathrm{hr}$ than at $0200 \mathrm{hr}$ (cf. Figs. $3 B$ and $6 B$ ).

Darkness period: 2000 to $0200 \mathrm{hr}$. From $1800 \mathrm{hr}$ to $0200 \mathrm{hr}$ the lamellar bodies remain in the same region of the A-segment but progressively lose volume, with lucent areas appearing in a few by $2000 \mathrm{hr}$ (Fig. 6D) and in the majority of the population by $2200 \mathrm{hr}$ (Fig. $6 E$ ). After $0200 \mathrm{hr}$ these structures shrink and without specific labeling become lost in the population of lysosomal organelles involved in the cycling of pigment granules and other structures in the A-segment.

Summary. Figure 7 summarizes the structural evolution of the early morning burst of photoreceptor membrane shedding through the 24-hr day. In early morning large quantities of microvillar membrane are organized into membrane whorls which balloon from the rhabdom into the R-segment cytoplasm. Membrane whorls give rise to a population of small, tightly assembled multivesicular bodies near the rhabdom which coalesce into large multivesicular bodies and move away from the eccentric cell dendrite toward the A-segment. Multivesicular bodies collapse into combination bodies as they reach the boundary of the R- and A-segments and continue to evolve into lamellar bodies, eventually appearing to lose material. The entire cycle is not complete by the end of $24 \mathrm{hr}$, but the ultimate fate of the shrinking lamellar bodies is obscured in these experiments by the abundance of similar organelles from other sources in the A-segment cytoplasm.

\section{Daily variations in total microvillar membrane area}

A ray of rhabdom is composed of two layers of abutting microvilli, one layer from each of two retinular R-segments.
The contact plane thus contains the circular ends of the abutting microvilli and has an array of open cylinders (the microvilli) normal to it on either side. We have determined the area of microvillar membrane in the array of microvilli attached to $1 \mu \mathrm{m}^{2}$ of contact plane. As described under "Materials and Methods," two types of measurements were made. The average length of microvilli was determined from light micrographs of $1-\mu \mathrm{m}$ transverse sections taken from the centers of ommatidia. The average area and perimeter of microvillar cross-sections were determined from electron micrographs of longitudinal sections. The average length, perimeter, and cross-sectional area were used to calculate the area of membrane for the average microvillus. The average cross-sectional area was used to determine how many microvilli were attached to the unit contact plane. The results below do not include the effects of any changes in the amount of surface area of a retinular cell $R$-segment which is specialized into rhabdomere.

Figure 8 shows the results of measurements and calculations for a 24 -hr period. During the burst of photoreceptor membrane shedding which occurs in early morning, the area of microvillar membrane per unit area of rhabdomere contact is reduced by about $70 \%$. Within an hour of the shedding burst, this area has returned to about $75 \%$ of the preburst value and $4 \mathrm{hr}$ after the burst is equal to the preburst value. The maximum amount of photoreceptor membrane per unit area of rhabdomere contact was achieved at three times during the day-just before the shedding burst, at $0800 \mathrm{hr}$, and at $1800 \mathrm{hr}$. Besides the major minimum at $0400 \mathrm{hr}$, secondary minima in the membrane area occurred at $1200 \mathrm{hr}$ and $2200 \mathrm{hr}$.

\section{Control mechanisms for photoreceptor membrane shedding}

\section{Light}

Light initiates the membrane shedding burst. In nature the daily burst of membrane shedding occurs at about $0400 \mathrm{hr}$ under very dim illumination and during ongoing efferent activity. What triggers the shedding burst is not clear from these data. To determine whether the shedding burst was triggered by light onset, animals were removed from natural lighting and placed in constant darkness in early evening. Starting at 2400 $\mathrm{hr}$, an animal was selected every $2 \mathrm{hr}$, exposed to $5 \mathrm{~min}$ of dim light (illuminance $=11$ lux , and fixed in dim light. Examination of the retinas showed that light onset initiated a burst of membrane shedding, as indicated by the breakdown of the rhabdom and formation of membrane whorls at each time. Thus, the first light onset can produce the daily shedding burst as early as midnight or as late as $2000 \mathrm{hr}$. At $0400 \mathrm{hr}$ the retina is still in a state of elevated sensitivity (Barlow, 1983). The ambient illumination must be sufficient to trigger the daily shedding burst since, if animals are maintained in the laboratory in absolute darkness, the shedding burst does not occur until the first exposure to light.

All photoreceptors shed membrane daily. Figure 9 shows light micrographs of arrays of ommatidia fixed at $0400 \mathrm{hr}$ and 1000 $\mathrm{hr}$. Note that at $0400 \mathrm{hr}$ all ommatidia show diminished rhabdoms due to membrane whorl formation. At $1000 \mathrm{hr}$ all ommatidia show multivesicular bodies moving toward the A-segment. Examination of the ommatidial arrays of many retinas reveals that the burst of photoreceptor membrane shedding occurs in every ommatidium each morning. Furthermore, the occurrence of the shedding burst is well synchronized across the retina.

Membrane shedding triggered by sudden light onset is similar to that triggered by gradual light onset. We have already outlined the sequence of structural changes that occur during and after the burst of membrane shedding triggered by natural dawn. Our original experiments (Chamberlain and Barlow, 1977, 1979), however, utilized sudden light onset. In those experi- 

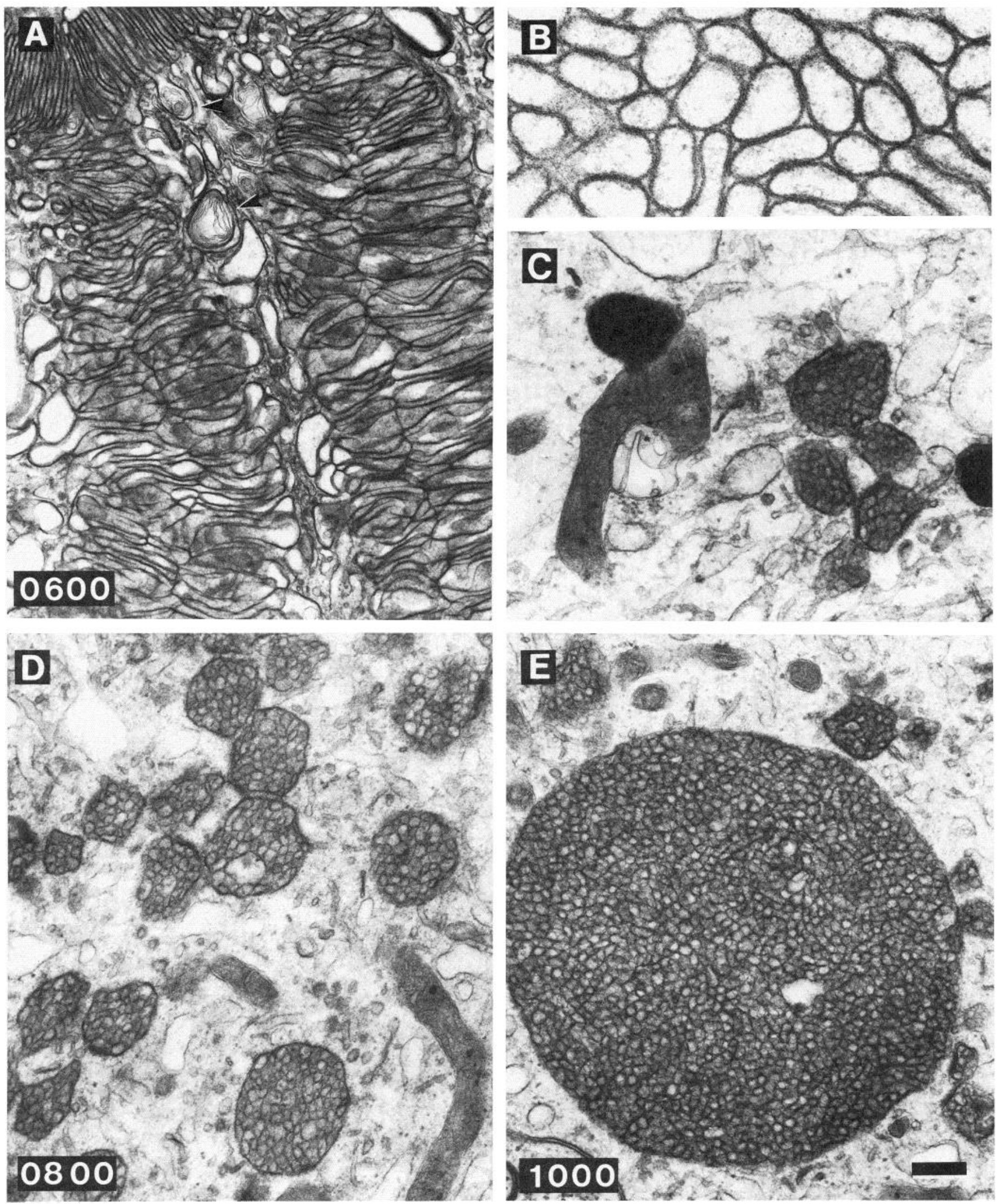

Figure 4. Ultrastructure of eyes fixed in midmorning. $A$ to $C$, By $0600 \mathrm{hr}$ the microvillar array has been restored. Whorls of membrane (arrowheads) distinct from those formed during transient membrane shedding are associated with the rhabdom now and throughout the day, but their significance is unclear. $B$, The microvillar diameters show more variation than at $0200 \mathrm{hr}$, but the fusion of adjacent membranes has been re-established. $C$, The cytoplasm of the R-segment contains many small tightly assembled multivesicular bodies which appear to be fusing. $D$, At $0800 \mathrm{hr}$ multivesicular bodies are arrayed in distinct bands parallel to the rhabom rays. $E$, By $1000 \mathrm{hr}$ multivesicular bodies have fused to form some large individuals which lie at the periphery of the R-segment near the junction with the A-segment. The bar in $E$ represents $0.56 \mu \mathrm{m}$ in $A, 0.1 \mu \mathrm{m}$ in $B, 0.24 \mu \mathrm{m}$ in $C$, and $0.3 \mu \mathrm{m}$ in $D$ and $E$. 

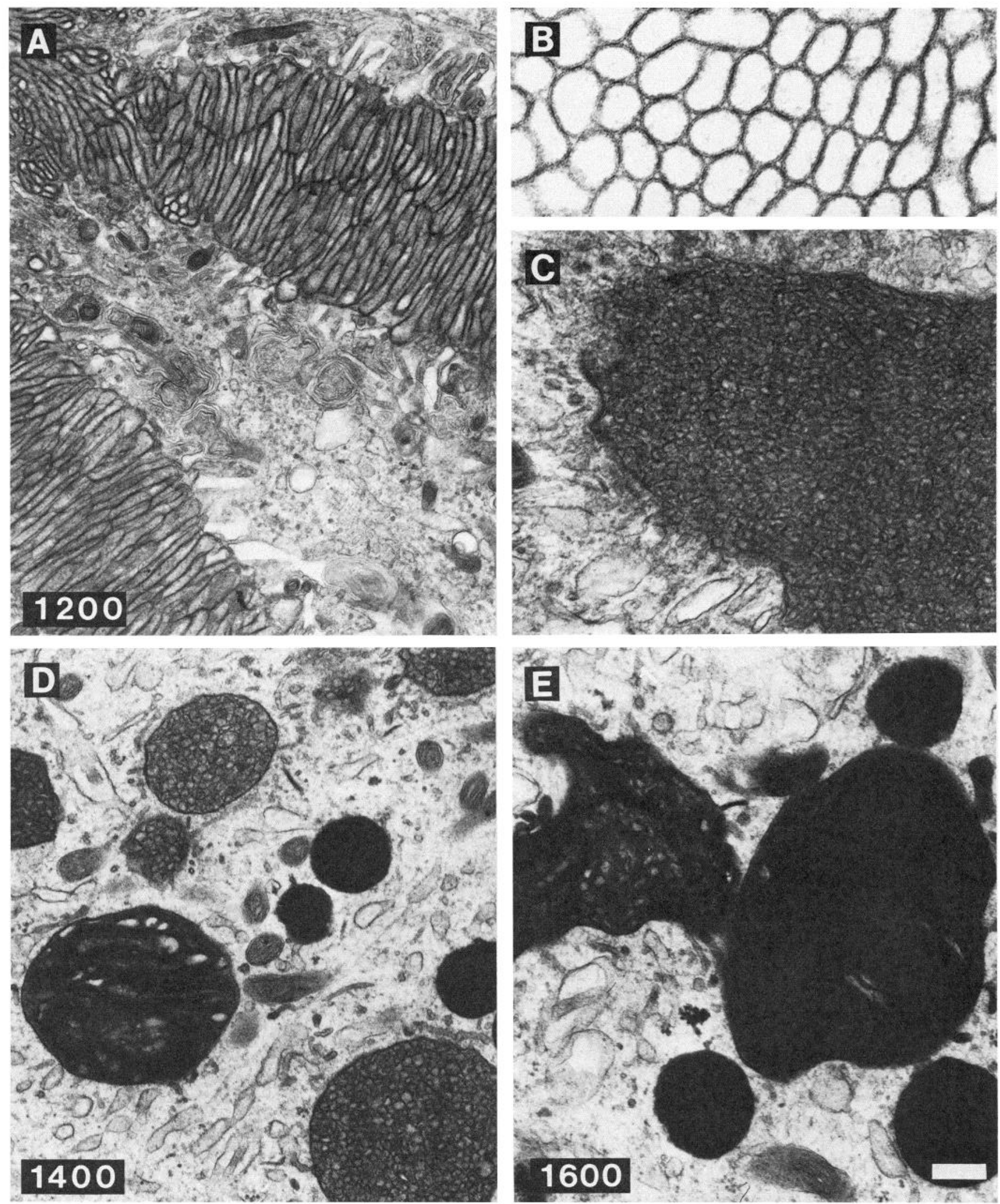

Figure 5. Ultrastructure of eyes fixed in the afternoon. $A$ to $C$, At $1200 \mathrm{hr}$ the rhabdom is prominently developed and well organized. $B$, The microvillar cross-sections show less variation than at $0600 \mathrm{hr}$. The fusion of adjacent membranes is well developed. $C$, Large multivesicular bodies at the junction of the R- and A-segments are somewhat collapsed relative to their appearance at $1000 \mathrm{hr} . D$, By $1400 \mathrm{hr}$ part of the population of multivesicular bodies has been converted to combination bodies by the formation of lamellar arrays. $E$, By $1600 \mathrm{hr}$ most of the multivesicular bodies have been transformed to combination bodies and many of these have further evolved to lamellar bodies with granular zones and lamellar zones. The bar in $E$ represents $0.56 \mu \mathrm{m}$ in $A, 0.1 \mu \mathrm{m}$ in $B, 0.24 \mu \mathrm{m}$ in $C$ and $E$, and $0.3 \mu \mathrm{m}$ in $D$. 

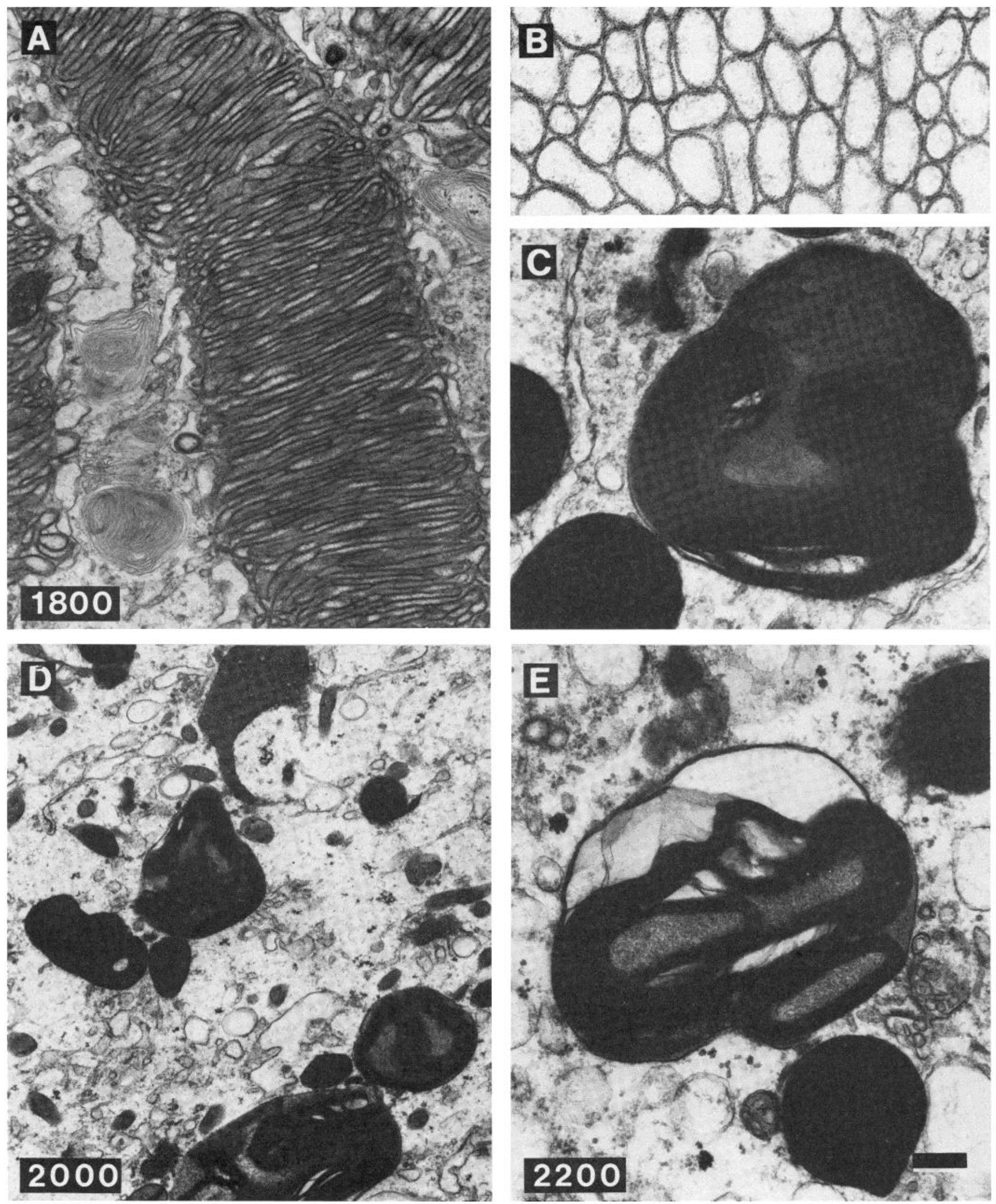

Figure 6. Ultrastructure of eyes fixed in the evening. $A$ to $C$, At $1800 \mathrm{hr}$, after exposure to light throughout the day, the rhabdom is still prominently developed and well organized. $B$, The fusion of adjacent membranes in the microvillar array is reduced relative to other times of the day. $C$, Lamellar bodies lie in the A-segment beyond the junction with the R-segment $D$, At $2000 \mathrm{hr}$ the lamellar bodies appear much the same as at $1800 \mathrm{hr}$ but have moved slightly further into the A-segment. $E$, By $2200 \mathrm{hr}$, the lamellar bodies begin to show empty areas and generally appear to be gradually losing material to the surrounding cytoplasm. The bar in $E$ represents $0.56 \mu \mathrm{m}$ in $A, 0.1 \mu \mathrm{m}$ in $B, 0.24 \mu \mathrm{m}$ in $C$ and $E$, and $0.6 \mu \mathrm{m}$ in $D$. 

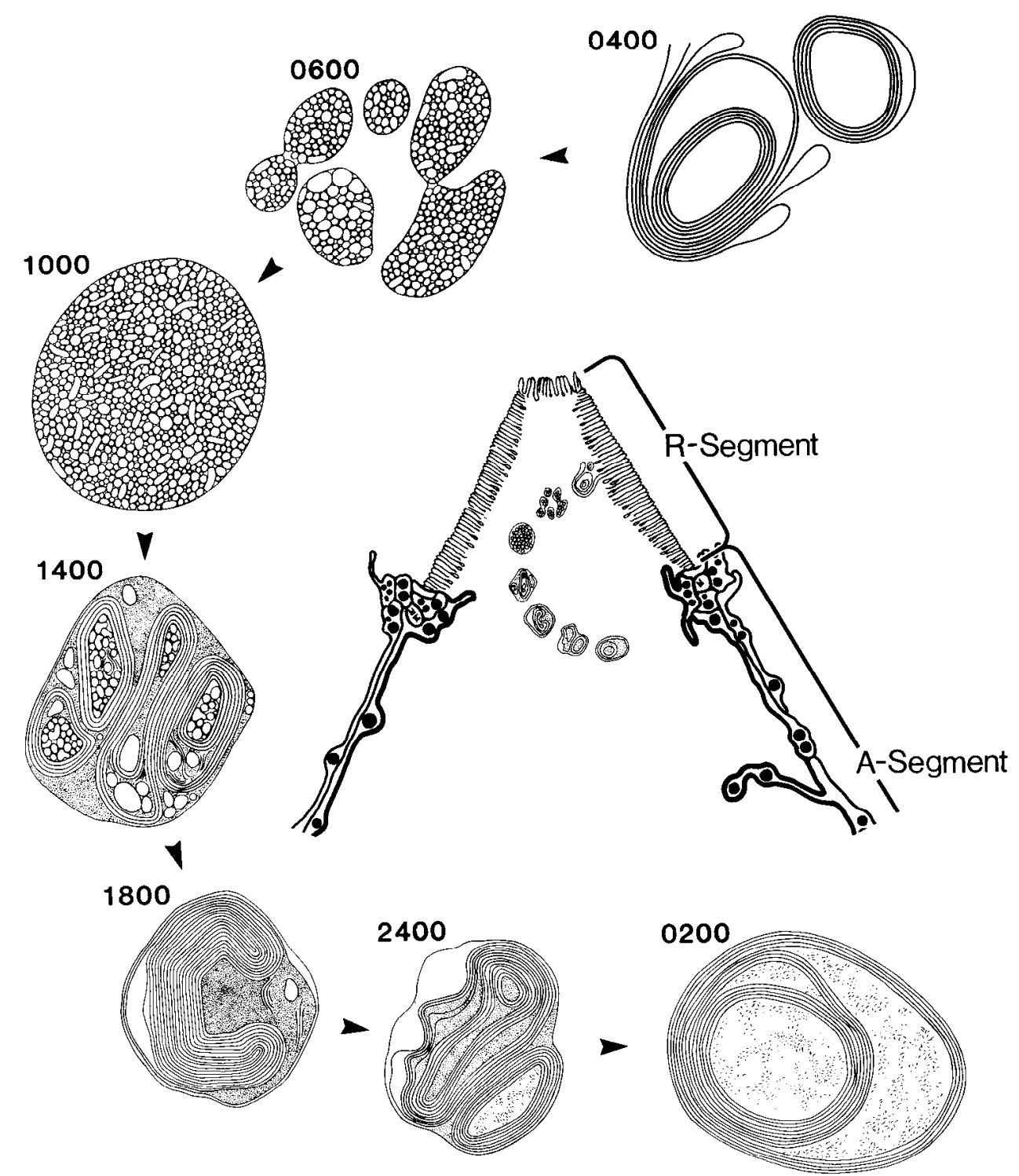

Figure 7. Summary of organelles involved in transient shedding of rhabdomeral membrane in Limulus. The drawing in the center shows the approximate positions of these structures in the retinular cell during the day (compare this with Figure 1). Whorls of microvillar membrane form in and adjacent to the rhabdom around $0400 \mathrm{hr}$. During the next $6 \mathrm{hr}$ these convert to multivesicular bodies and fuse to form large multivesicular bodies near the periphery of the R-segment ( $1000 \mathrm{hr}$ ). Through the afternoon the multivesicular bodies collapse to combination bodies (1400 hr) and ultimately to lamellar bodies $(1800$ to $0200 \mathrm{hr})$. During this process they move further into the A-segment where they become lost in the general population of residual bodies.

ments we varied the times of light onset and fixed all animals at the same time of day ( $1600 \mathrm{hr})$. We observed no significant differences in the membrane whorls occurring during shedding nor in the succession of lysosomal organelles that follows. Thus, the structural sequence of the shedding process is the same whether it is triggered by sudden light onset or the gradual onset of natural dawn.

Is the time course of transient membrane shedding the same for both sudden and gradual light onsets?

Does the time course of transient membrane shedding depend upon whether the light trigger is gradual or sudden? Figure 10 shows the average microvillar length, as a function of time in the light, for animals exposed to sudden light at staggered times and fixed at $1600 \mathrm{hr}$ (Fig. 10, solid line). Exact specification of the temporal progression of membrane shedding is difficult for animals exposed to natural lighting because of the uncertainty about the time of occurrence of the shedding burst. We have matched the time of the shedding burst in the data from Figure $13 \mathrm{~A}$ for animals fixed under natural conditions and have replotted these data as time after the start of transient shedding (Fig. 10, dashed line). The minimum length of microvilli, achieved during the peak of membrane shedding, is nearly the same for these two experiments. Although the changes in the 8 $\mathrm{hr}$ after the initiation of the shedding burst are different in magnitude, within the uncertainty of our measurements, they are not significantly different in trend whether the burst is triggered by natural dawn or sudden light onset.

Experiments using sudden light onset have allowed us to investigate early stages of transient shedding. Figure 11 shows the earliest stages in the process of transient membrane shedding which we have been able to produce with sudden light onset. Without light exposure, the rhabdom is well organized with prominent subrhadomeral palisades. Although the palisades remain well organized, light exposure during fixation is apparently able to trigger the formation of membrane whorls since our double aldehyde fixative requires some time to crosslink proteins in the tissue. Fifteen minutes of light exposure before fixation is sufficient to induce the complete production 


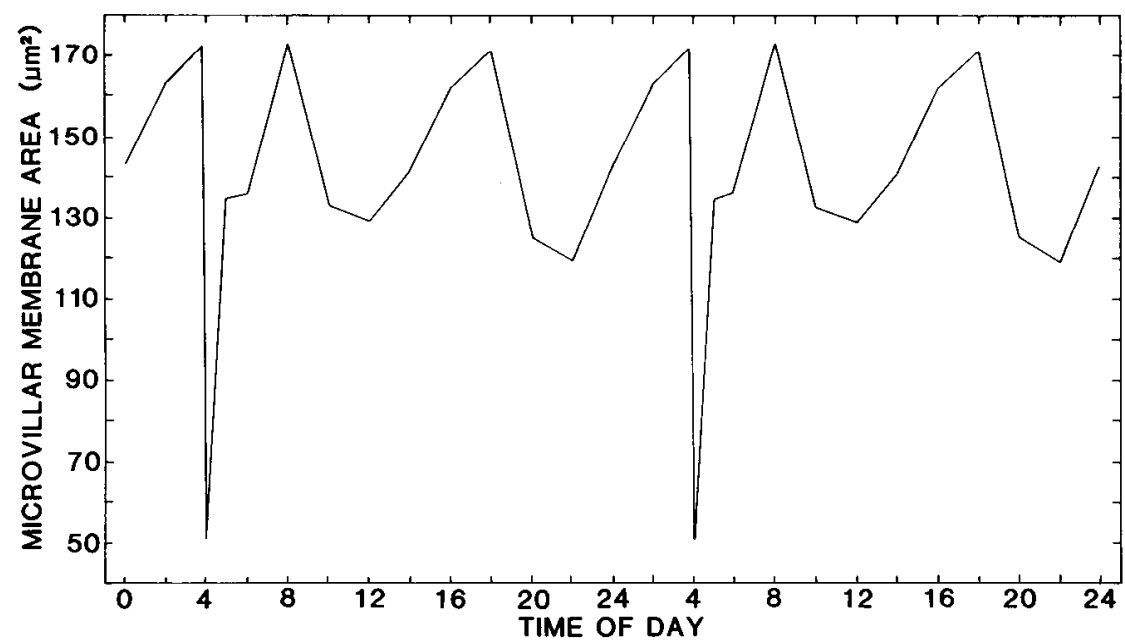

Figure 8. Daily variation in microvillar membrane area in the Limulus lateral cye. The ordinate shows the total area of microvillar membrane in the rhabdom of two retinular cells attached to a $1-\mu \mathrm{m}$ square in their plane of contact (e.g., the plane of Fig. $3 B$ ). The marked reduction in area at $0400 \mathrm{hr}$ represents the result of the formation of membrane whorls during transient membrane shedding. Otherwise there is no strong correlation between the amount of photosensitive membrane and the light and dark portions of the diurnal cycle. These values have been calculated from measured mean microvillar lengths presented in Figure 13A and the following measured microvillar cross-sectional areas (timemean $\left.\left(\mu \mathrm{m}^{2}\right) \pm \mathrm{SD}\right): 0200,0.0073 \pm 0.003 ; 0400,0.034 \pm 0.021 ; 0600,0.009 \pm 0.004 ; 0800$, $0.0048 \pm 0.0017 ; 1000,0.0075 \pm 0.002 ; 1200,0.0065 \pm 0.002 ; 1400,0.0057 \pm 0.0025 ; 1600$, $0.0056 \pm 0.0017 ; 1800,0.0061 \pm 0.0015 ; 2000,0.0103 \pm 0.0052 ; 2200,0.0070 \pm 0.002 ; 2400$, $0.0071 \pm 0.002$. Data for one $24-\mathrm{hr}$ period have been plotted twice to show the full daily pattern without breaks.

of membrane whorls. Both the triggering mechanism and the process of membrane whorl formation appear to be relatively rapid.

\section{Efferent input}

Efferent optic nerve activity from a circadian clock primes transient membrane shedding. As we reported earlier (Chamberlain and Barlow, 1979), not only does the daily burst of membrane shedding require light for initiation, but it also requires priming by a period of efferent input to the retina. Cutting the optic nerve and thereby removing efferent input to the retina abolishes the light-initiated shedding burst. Shocking the peripheral stump of the cut optic nerve during the night to mimic normal efferent activity restores the light-initiated shedding burst.

Further investigation revealed that blocking efferent input by cutting the optic nerve does indeed abolish rather than delay or diminish the daily burst of membrane shedding. As a more extensive test of our earlier results, we cut both lateral optic nerves of a group of animals. After 2 weeks in natural lighting, these animals were sacrificed under the same conditions as the normal animals used for the natural-lighting data described above. None of the organelles associated with transient photoreceptor membrane shedding were observed in the ommatidia of any of the animals with chronically cut lateral optic nerves. Figure 12 shows two examples. In both cases the residual organelles (Fig. 12, arrows) are absent from the animals with cut optic nerves.

Three to $5 \mathrm{hr}$ of efferent input primes shedding. How much efferent activity is required to prime the shedding burst? Animals were removed from natural lighting at $1800 \mathrm{hr}$ and placed in total darkness. Two animals were selected, exposed to dim light (illuminance $=11$ lux) for $5 \mathrm{~min}$, and fixed at $2200 \mathrm{hr}$ and every $2 \mathrm{hr}$ thereafter for $24 \mathrm{hr}$. Light did not initiate a burst of membrane shedding at $2200 \mathrm{hr}$ but did initiate the burst at $2400 \mathrm{hr}$ and at all subsequent sample times. Since the onset of efferent activity in the evening is gradual over about an hour and may not occur at precisely the same time for all animals, we can only estimale that between 3 and $5 \mathrm{hr}$ of efferent input is required to prime transient membrane shedding. Since in nature shedding occurs at dawn, $8 \mathrm{hr}$ or more after onset of efferent activity, the timing of the shedding burst is apparently determined by the ambient light level rather than the duration of efferent input from the circadian clock.

Shedding occurs only once a day. Light onset will apparently initiate only one shedding burst per day. If an animal maintained in natural light is placed in darkness in midmorning and exposed to light and fixed in late afternoon, no shedding burst is initiated, although the multivesicular bodies and combination bodies resulting from the shedding burst at dawn are present in the cytoplasm. This observation is consistent with the requirement for efferent priming to precede the shedding burst.

Rhabdomeral membrane area undergoes changes that are independent of efferent activity. Besides the pronounced decrease in microvillar membrane area during the early morning burst of membrane shedding, Figure 8 shows a rather complex variation in membrane area over the 24 -hr light/dark cycle. Is this variation significant? To investigate this question we measured the mean microvillar length for the population of animals with both optic nerves cut to block circadian efferent input to the retina. Figure 13 shows the average microvillar length over the 24-hr period of natural lighting for the two groups. Note that, although the length is generally shorter and the early morning shedding burst is absent in animals with cut optic nerves, the overall pattern is noticeably similar under the two conditions. This variation represents a diurnal rhythm in the length of microvilli that is independent of efferent activity from the circadian clock.

\section{Discussion}

Control mechanisms for membrane shedding in Limulus. Early each morning the structure of the photosensitive rhabdom of each lateral eye ommatidium is interrupted by a massive burst of membrane shedding. This shedding burst is primed by efferent input from a circadian clock in the brain but must be initiated by light. Only 3 to $5 \mathrm{hr}$ of efferent input are required 

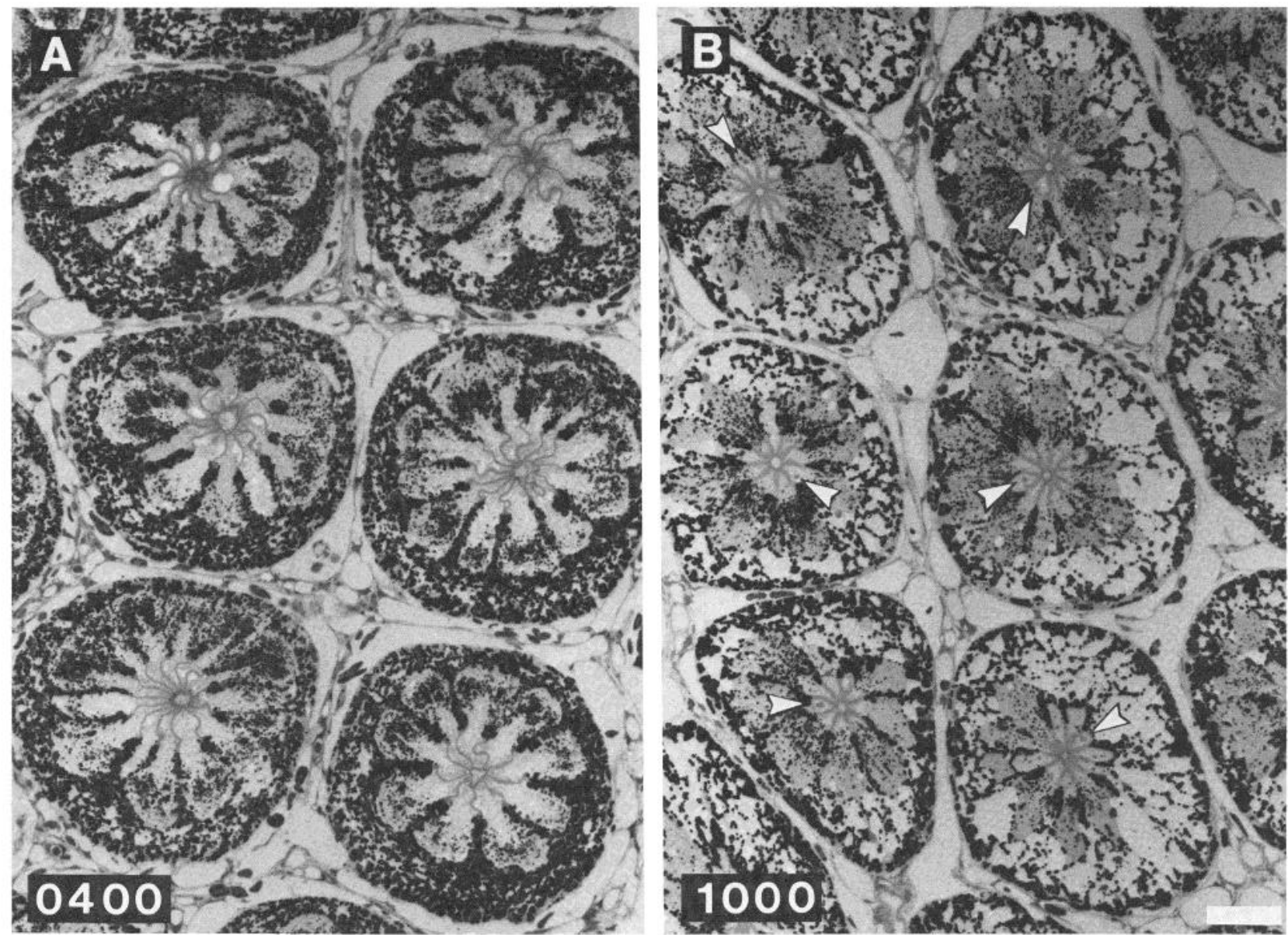

Figure 9. Synchrony of photoreceptor membrane shedding across the retinal array. $A$ shows several ommatidia from the lateral eye of an animal fixed at $0400 \mathrm{hr}$. The rhabdoms of all ommatidia are attenuated from the simultaneous formation of membrane whorls. $B$ shows a similar view from the eye of an animal fixed at $1000 \mathrm{hr}$. The arrowheads mark large multivesicular bodies present in the cytoplasm which are visible in each ommatidium. The synchrony of early morning membrane shedding across the retinal array is relatively good. The $b a r$ represents $52 \mu \mathrm{m}$.

Figure 10. Comparison of the time course of transient membrane shedding under natural lighting and laboratory conditions of sudden light onset. The solid line gives the mean microvillar length $\pm \mathrm{SD}$ for animals exposed to sudden light onset at staggered times and all fixed at $1600 \mathrm{hr}$. The dashed line gives mean microvillar length for animals fixed under ambient natural light. For the dashed line the data have been plotted relative to transient membrane shedding at $0400 \mathrm{hr}$ ). Although the magnitudes are different, the general trends of the data are the same under these two conditions.

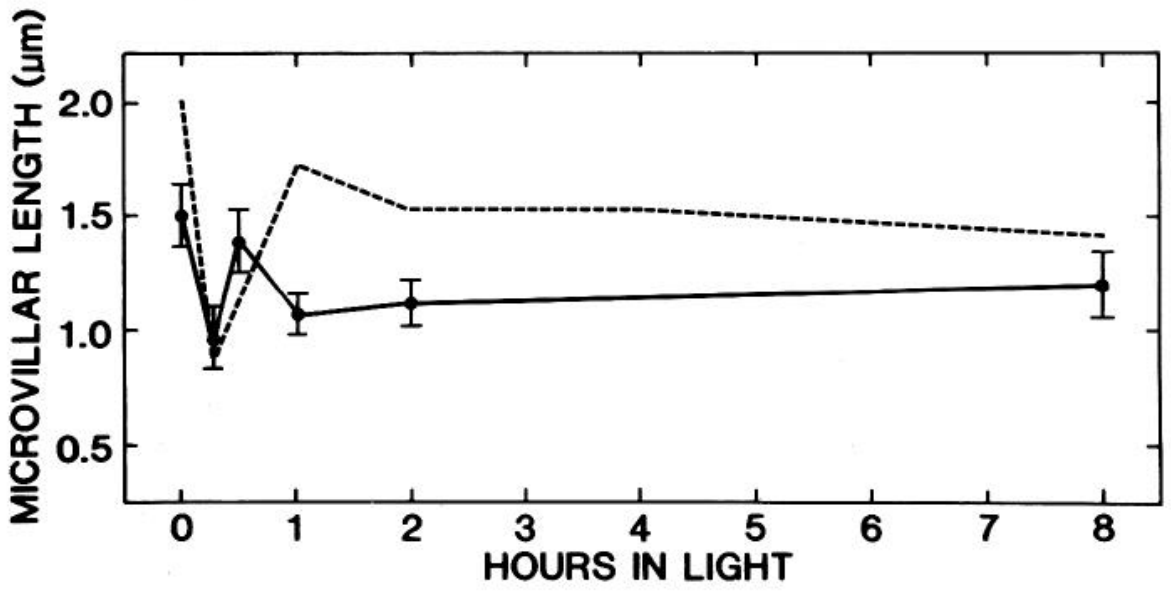

for priming so that, under natural lighting, it is the onset of light at dawn which controls the timing of the burst. Despite the very gradual onset of light during natural dawn, the shedding burst is synchronous across each retinal array, suggesting that the initiation of the shedding burst may ultimately be controlled by a property of the whole retinal array rather than a property of an individual ommatidum.

Shedding begins with the formation of whorls of microvillar membrane which give rise to an orderly sequence of lysosomal organelles that gradually move away from the rhabdom and migrate peripherally to the A-segment of the photoreceptor. The disorganization of the rhabdom by the formation of membrane whorls is short-lived, however, and as soon as $30 \mathrm{~min}$ after light onset, the microvillar array is again well organized, with essentially as much membrane area as before the shedding burst. The mechanisms by which the rhabdom is so rapidly reconstructed are unknown.

The membrane shedding burst is not the sole mechanism involved in the degradation of photosensitive membrane. At other times of the day there are gradual reductions and in- 

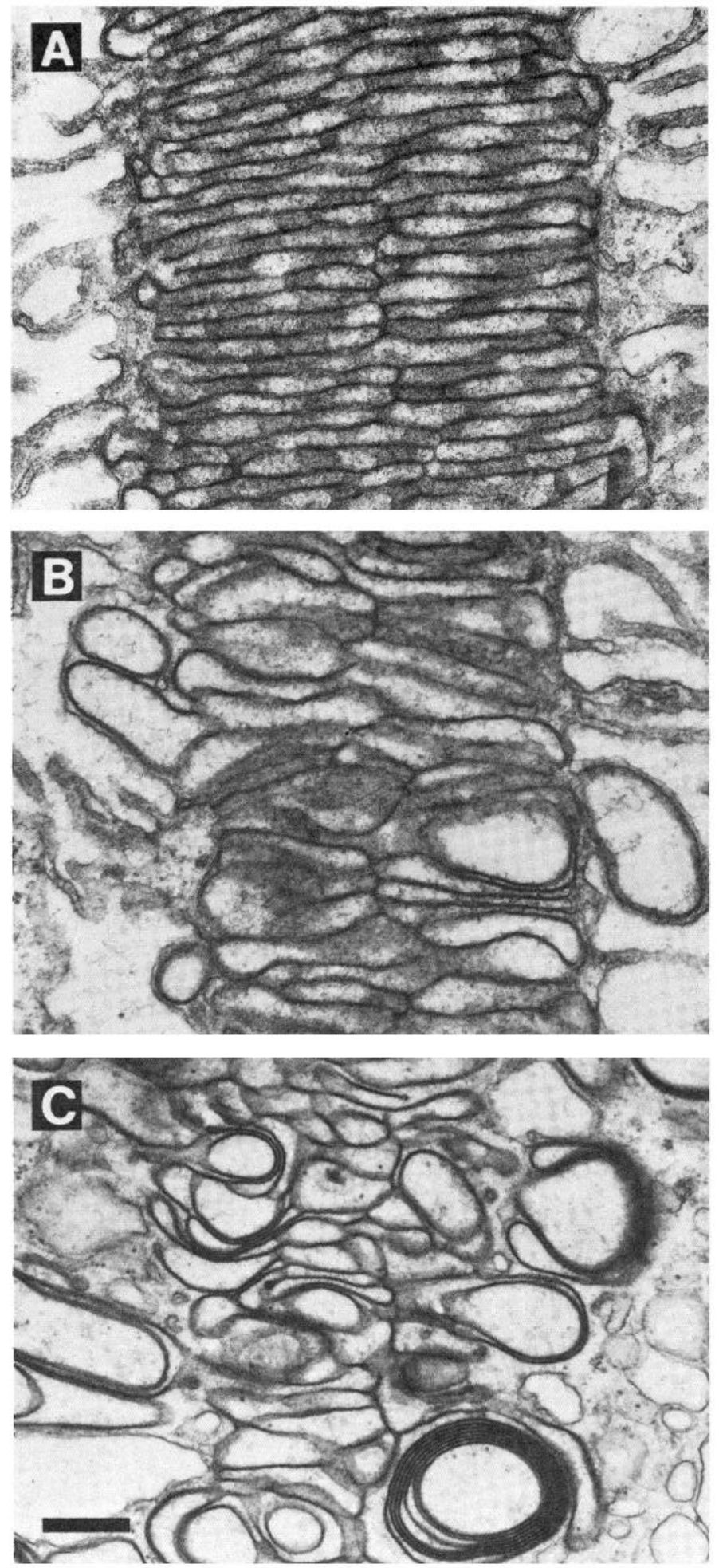

Figure 11. Light initiation of transient membrane shedding in the Limulus lateral eye. A, Ultrastructure of the rhabdom of an animal maintained overnight in complete darkness and fixed in complete darkness. $B$, Ultrastructure of the rhabdom of an animal maintained overnight in complete darkness, injected with fixative in complete darkness, and then exposed to light. $C$, Ultrastructure of the rhabdom of an animal maintained overnight in complete darkness, exposed to light for $15 \mathrm{~min}$, and fixed in light. All three animals were fixed at noon. The bar represents $0.5 \mu \mathrm{m}$. creases in the size of the rhabdom which do not require efferent input. This suggests additional mechanisms of membrane shedding and synthesis that are not under efferent control. The details of these mechanisms and their controlling factors remain to be investigated.

Formation of membrane whorls and tightly assembled multivesicular bodies. Our results indicate that the light onset triggers a rapid series of events which involves a breakdown in the structural integrity of the rhabdom. Efferent input from the circadian clock in some way sets up a structural instability in the microvillar array. Light, either directly or indirectly, triggers the expression of this instability. Once the instability has been triggered, the quintuple-layered membranes between microvilli break apart, and the microvilli themselves largely collapse into membrane whorls.

We do not understand the details of the mechanism by which the microvillar arrays are transformed to membrane whorls. Figure 14, $A$ and $B$, suggests that microvilli fuse to form closed discs of membrane in parallel stacks that fold into spherical whorls of membrane (Fig. 14A). The volume within the whorl is filled with cytoplasm as evidenced by the occasional entrapment of a mitochondrion or retinular pigment granule within a membrane whorl (Fig. 14B). Note that only under laboratory conditions of sudden light onset are pigment granules in the Rsegment during the formation of membrane whorls. In nature they are dispersed in the peripheral A-segment in early morning when transient shedding occurs.

Blest et al. (1978) described the two types of multivesicular bodies resulting from membrane shedding in the spider Dinopis as loosely and tightly assembled. We are using their terminology to describe a similar, but not identical dichotomy. In Dinopis, loosely assembled multivesicular bodies result from the assembly of coated vesicles, that form at the base of rhabdomeral microvilli, into membrane-bound multivesicular bodies, probably by secondary endocytosis (Eguchi and Waterman, 1976). Tightly assembled multivesicular bodies form from the adhesion of smooth vesicles which result from the breakup of whorls of rhabdomeral membrane and are not initially membrane bound. In Limulus, similar loosely assembled multivesicular bodies are present in the cytoplasm. However, we do not know whether these bodies are involved with the daily transient shedding of rhabdomeral membrane (see discussion below). Tightly assembled multivesicular bodies result from the breakup of membrane whorls but, unlike those of Dinopis, appear to be membrane bound even in the smallest sizes. We do not completely understand the transformation from membrane whorl to multivesicular body, but Figure $14, D$ and $E$, shows what may be intermediate stages. The conversion does not appear to involve coated vesicles, but rather the formation of tubular extensions from membrane whorls. These somehow give rise to short segments of tubule with one to several vesicles inside. Progressive fusion of vesiculated tubules produces multivesicular bodies. The initial stages are not so tightly assembled as later stages and have some of the characteristics of loosely assembled multivesicular bodies (Fig. $14 E$ ), but as they enlarge, they become more densely packed (Fig. 4). At no stage do they closely resemble the other sort of loosely assembled multivesicular body we observe (Fig. 15B).

Membrane synthesis. We have not been able to clearly identify synthesis organelles that might provide for the reconstitution of the microvilli after the obvious disorganization which occurs as part of the process of shedding. The studies of rapid rhabdom synthesis by Stowe (1980) and Kataoka and Yamamoto (1983) suggest another interpretation for the membrane whorls we see in Limulus. During the rapid assembly of microvilli in crab photoreceptors, membrane whorls very similar in appearance to those which accompany the burst of membrane 

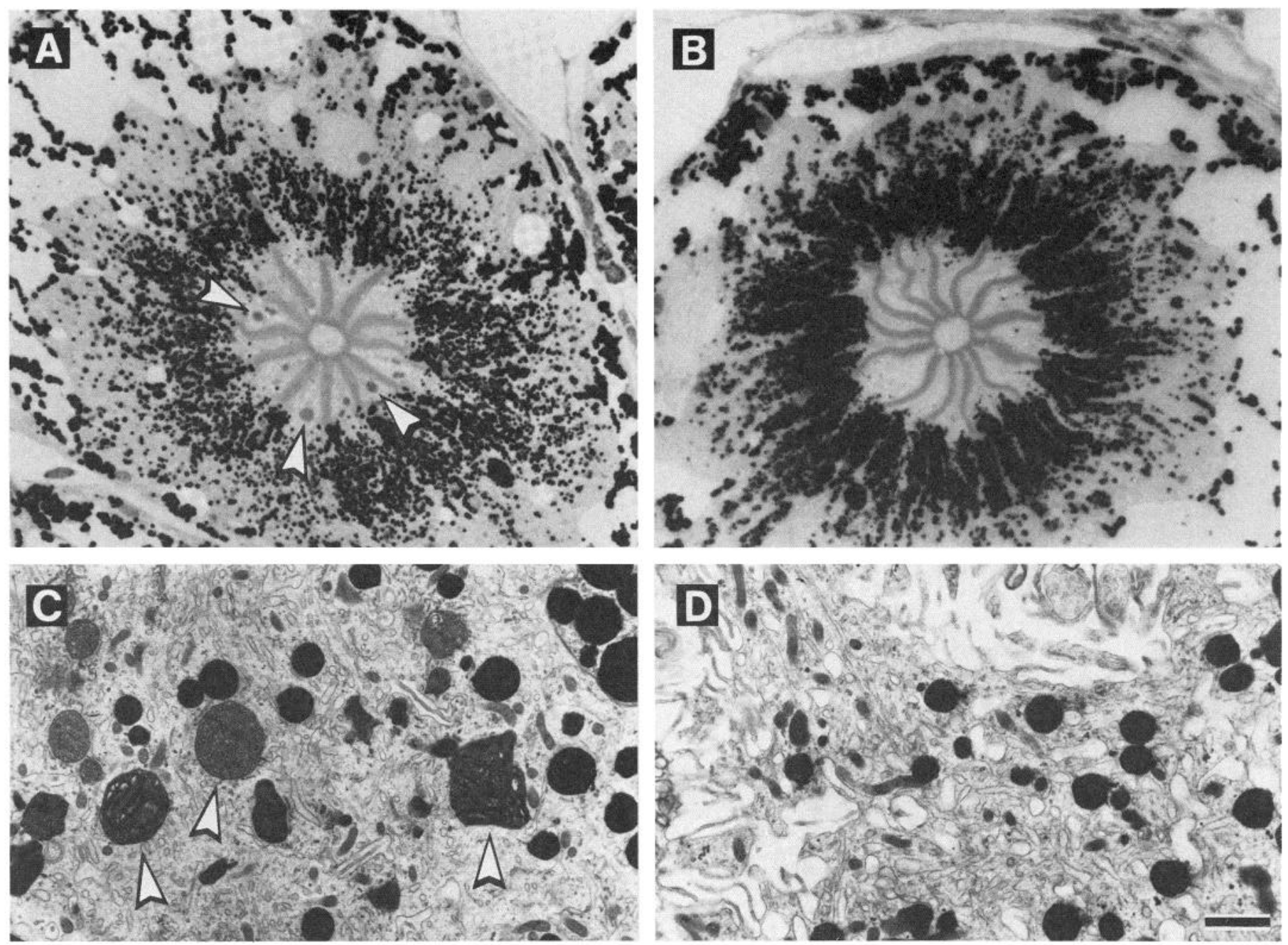

Figure 12. Priming of transient membrane shedding by efferent inputs. A, In intact animals maintained in natural lighting, multivesicular bodies (arrowheads) from transient membrane shedding around $0400 \mathrm{hr}$ are visible with the light microscope at $1000 \mathrm{hr}$. B, In animals with chronically removed efferent input maintained in natural lighting, no multivesicular bodies are visible in the retinular cell cytoplasm. $C$, At 1400 $\mathrm{hr}$, intact animals maintained in natural lighting show a variety of multivesicular and combination bodies (arrowheads) in the retinular cell cytoplasm near the junction between the $\mathrm{R}$-segment and A-segment. $D$, Animals with chronically removed efferent input show no such structures at $1400 \mathrm{hr}$. Removing efferent input blocks the formation of membrane whorls at $0400 \mathrm{hr}$ and all subsequent organelles associated with transient membrane shedding. The $b a r$ in $D$ represents $18 \mu \mathrm{m}$ in $A$ and $B$, and $1 \mu \mathrm{m}$ in $C$ and $D$.

shedding in Limulus are involved in the addition of membrane to the microvillar array. Our measurements of microvillar area before and after the shedding burst (Fig. 8) indicate that nearly as much membrane exists after the burst as before. Perhaps the membrane whorls are not membrane on the way out, but membrane on the way in.

The rapid increase of the rhabdom membrane area to essentially its preburst size without clear evidence of membrane synthesis raises questions about the nature of the shedding burst itself. What is the material that ultimately ends up in the multivesicular bodies? Does the process of whorl formation involve the selective removal of a specific component of the rhabdom membrane? Is some worn-out component of the membrane stripped off and packaged in the multivesicular bodies? If so, how is this component ultimately replaced in the rhabdom? The answers to these and other questions are not yet known.

Other organelles in the $R$-segment. We have outlined the sequence of organelles associated with transient shedding of rhabdom membrane. At first light onset, microvillar membrane is organized into membrane whorls which give rise, in sequence, to tightly assembled multivesicular bodies, combination bodies, and lamellar bodies. Except for variations in the initial stages of microvillar breakdown, this sequence matches well that found in other invertebrate species such as mosquito (White, 1968), shrimp (Itaya, 1976 a, b), dinopid spiders (Blest, 1978), crayfish (Hafner et al., 1980), and blowfly (Williams, 1982a). In addition to those structures involved in this sequence, we also observed organelles in the R-segment in the vicinity of the rhabdom whose role in cycling of photoreceptor membrane is less certain.

Figure 15 shows examples of organelles whose function, if any, in membrane tunover is uncertain. Loosely assembled multivesicular bodies (Fig. 15B) are found in most of these systems. They are bound by membranes which appear to be derived from endoplasmic reticulum (Blest et al., 1978) and generally contain smooth vesicles which probably form by secondary endocytosis from extravacuolar coated vesicles (Eguchi and Waterman, 1967, 1976; White, 1968; Blest et al., 1978). In mosquito and crayfish, for example, the principal pathway 

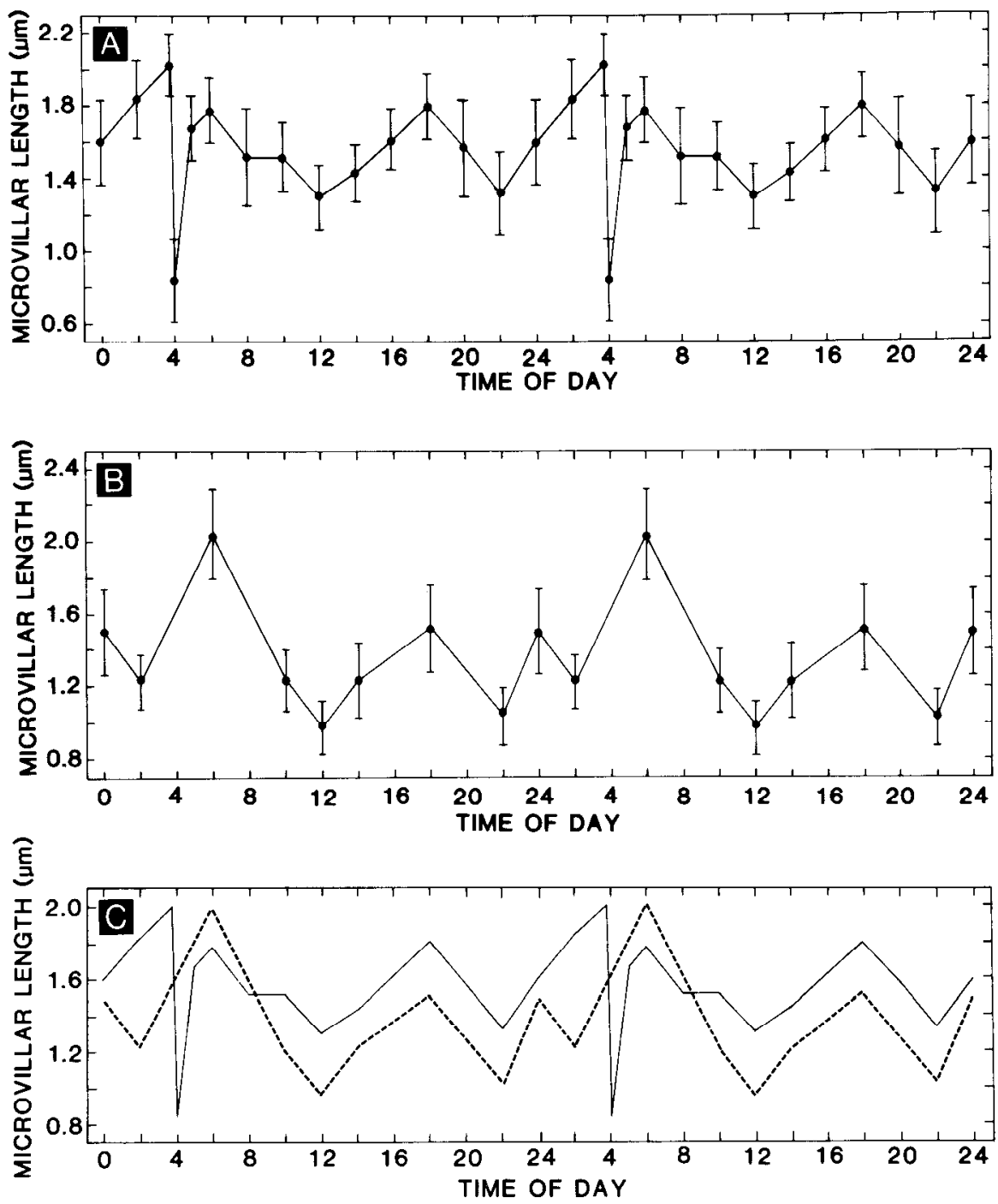

Figure 13. Morphometric analysis of microvillar length in natural lighting in the presence and $a b-$ sence of efferent inputs. $A$, Mean microvillar lengths $\pm \mathrm{SD}$ for animals maintained and fixed in natural lighting. The dip at $0400 \mathrm{hr}$ (4:00 A.M.) represents the reduction in microvillar length due to the formation of membrane whorls during transient membrane shedding. $B$, Mean microvillar lengths \pm SD for animals with chronically removed efferent input, maintained and fixed in natural lighting. The sharp dip at $0400 \mathrm{hr}$ is absent, reflecting the blocking of transient membrane shedding. $C$, The curves from $A$ (solid) and $B$ (dashed) plotted on the same coordintes. Except for the sharp dip at $0400 \mathrm{hr}$, the pattern of the two curves is similar, reflecting cyclic changes in the size of the rhabdom that are not dependent on the activity of retinal efferents. One 24 -hr cycle of data has been plotted twice to avoid breaks in the pattern. of rhabdom membrane shedding is by the formation of coated vesicles and loosely assembled multivesicular bodies (White, 1968; Eguchi and Waterman, 1976; Hafner et al., 1980). By contrast, Blest et al. (1978) have suggested that tightly assembled multivesicular bodies result from the direct conversion of membrane whorls associated with large-scale rhabdom shedding. They found that both tightly assembled and loosely assembled types are produced during the dawn breakdown of the rhabdom in Dinopis. In Limulus we found that loosely assembled multivesicular bodies are not specifically associated with the early morning burst of membrane shedding. Rather, they, and their precursor coated vesicles, are seemingly always present in low numbers during the 24-hr cycle. Perhaps loosely assembled multivesicular bodies are involved in the shedding of 'rhabdom, which occurs in the absence of circadian efferent input, or in the breakdown of some other cellular component.

Whorls of membrane that are markedly different from those of transient shedding are common in the $\mathrm{R}$-segment during much of the day (Fig. 15A). Other membrane-bound collections of membrane whorls and tubules are also commonly present (Fig. 15, $C$ and $D$ ). Often these organelles appear to be associated with the microvilli of the rhabdom (Figs. $4 A$ and 15, $C$ and $D$, arrowheads), but whether they represent membrane shedding or membrane synthesis is unclear-the daily variation in the extent of the rhabdom (Fig. 13) implies that both processses occur during various times of the day. Since these structures persist in the chronic absence of efferent input, they are likely to be part of the underlying diurnal rhythm in rhabdom structure (Fig. 13B).

Fixation artifacts. Evidence has recently been presented which suggests that the membrane whorls observed during transient membrane shedding might be an artifact of fixation (Williams, 1980; White and Michaud, 1981; Blest et al., 1982). The trend of these findings is that divalent cations, particularly calcium ions, in the fixative or prolonged aldehyde fixation before osmication may produce whorls of membrane in microvillar arrays, especially in membrane ready to be shed. Whether or not the membrane whorls we observe are induced by fixation is not critical to the main points of the studies reported here. Rather, the unique association of membrane whorls with the onset of membrane shedding makes them a particularly useful marker in our investigations of the control mechanisms for membrane shedding.

Nonetheless, our control experiments suggest that the membrane whorls may be quite resistant to changes in fixation procedure. Using our standard fixative, but shortening the fixation time to $1 \mathrm{hr}$, as suggested by Williams (1980), produces a less well preserved cytoplasmic background but does not affect the appearance of the whorls. Tissue fixed in the standard way with $2 \mathrm{~mm}$ EGTA (Williams, 1980) added to the standard fixative is virtually indistinguishable from tissue fixed without EGTA. The addition of $5 \mathrm{~mm}$ calcium chloride to our standard fixative prepared in cacodylate buffer changes the appearance to the cytoplasm but does not affect the appearance of the 

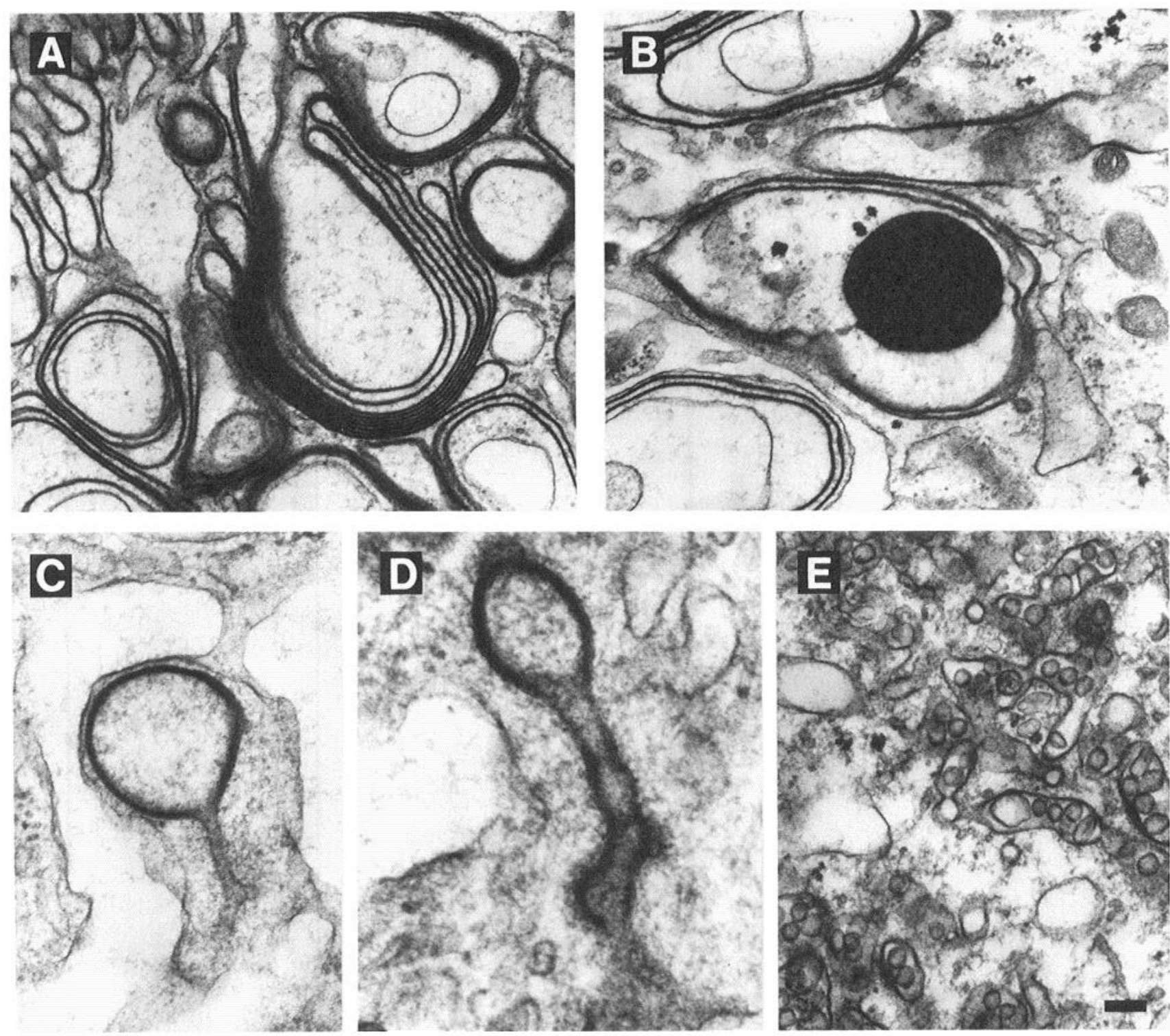

Figure 14. Formation of membrane whorls and conversion to multivesicular bodies. A, Membrane whorl formed 15 min after light onset. The concentric membrane profiles result from arrays of closed profiles of microvillar membrane. $B$, Membrane whorl surrounding pigment granule. Sudden light onset causes pigment granules to enter the R-segment where they and mitochondria are occasionally trapped within membrane whorls. The center of a whorl is thus cytoplasm. $C$ and $D$, Possible conversion of whorl to vesicles. Such structures are observed $1 / 2$ hr and 1 hr after light onset and may represent the intermediate between membrane whorls and multivesicular bodies. $E$, Very early stages of multivesicular bodies. Even when the multivesicular bodies contain only a few vesicles they are still membrane bound. The bar in $E$ represents $0.2 \mu \mathrm{m}$ in $A$ and $B, 0.1 \mu \mathrm{m}$ in $C, 0.05 \mu \mathrm{m}$ in $D$, and $0.06 \mu \mathrm{m}$ in $E$.

membrane whorls. Fixation in $40 \%$ osmium tetroxide in carbon tetrachloride does not preserve the cytoplasm but does preserve the appearance of the membrane whorls. The formation of membrane whorls in the Limulus rhabdom would not appear to be a function of calcium in the fixative, of calcium fluxes caused during fixation, or of prolonged fixation.

Comparison with earlier work on Limulus. Behrens and Krebs (1976) reported that the size of the rhabdom is significantly reduced when the animal is exposed to prolonged periods of light. As shown in Figure 13A, the rhabdoms of intact animals exposed to normal diurnal lighting undergo complex variations in size during a $24-\mathrm{hr}$ period. Whether a dark- or light-adapted rhabdom is large or small depends critically upon when in the diurnal cycle the animal is examined. The additional complexity introduced when an artificial regimen of lighting is superimposed on the underlying circadian rhythms in visual function
(Barlow, 1983) makes data such as those presented by Behrens and Krebs (1976) particularly difficult to interpret. We suspect that the strong diminution of the rhabdom observed by these authors may have resulted from periods of light which extended into the late evening when the circadian clock was active, and when the animal would normally be in darkness.

Control mechanisms for membrane shedding: Invertebrates. We have shown in Limulus that transient membrane shedding is initiated by light and requires efferent input from a circadian clock in the brain. In other vertebrates photoreceptor membrane shedding also appears to be induced by light-e.g., shrimp (Itaya, 1976a, b), mosquito (White, 1967), crayfish (Eguchi and Waterman, 1967; Hafner et al., 1980), locust (Williams, 1982b), crab (Nässel and Waterman, 1979), spider (Blest, 1978), and abalone (Kataoka et al., 1982). Some studies with invertebrates have shown evidence of an underlying endogenous control 

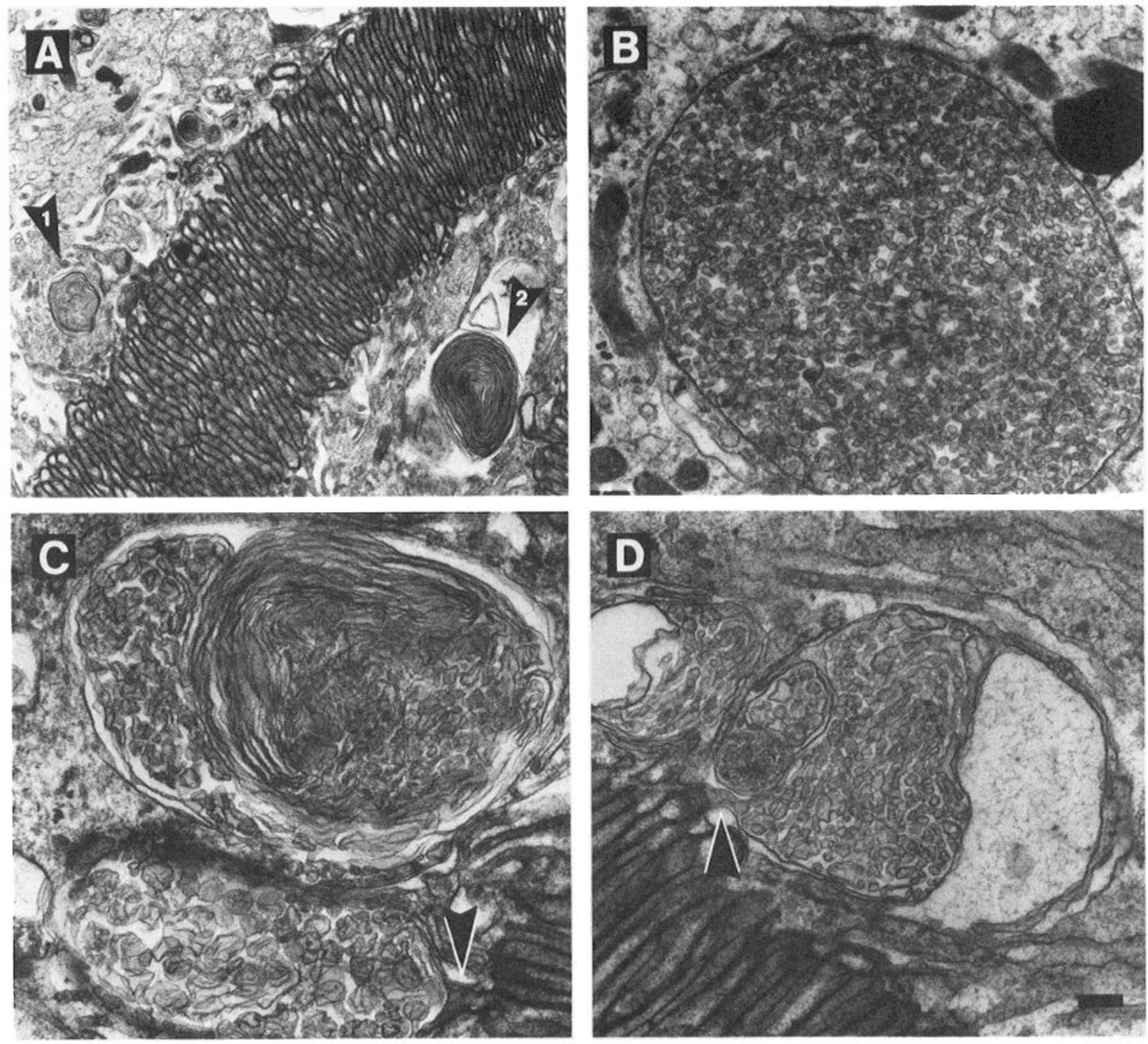

Figure 15. Other organelles found in the R-segment. A, Loosely aggregated masses of membrane tubules (arrowhead 1) and tight whorls of membrane (arrowhead 2) are common in small numbers throughout the day. Whether these are associated with membrane shedding or synthesis is unclear. $B$, Loosely assembled multivesicular bodies are found throughout the day and are clearly not associated with the progression of organelles resulting from transient membrane shedding. $C$ and $D$, Membrane-bound assemblages of membrane tubules and lamellae are often observed to connect to the microvillar array (arrowheads). What roles, if any, these structures play in membrane shedding or synthesis is unclear except that they are present whether or not the early morning transient shedding event occurs. The bar in $D$ represents $0.48 \mu \mathrm{m}$ in $A, 0.24 \mu \mathrm{m}$ in $B, 0.19 \mu \mathrm{m}$ in $C$, and $0.19 \mu \mathrm{m}$ in $E$.

mechanism-e.g., crab (Nässel and Waterman, 1979), locust (Horridge et al., 1981; Williams, 1982b), and spider (Blest, 1978). Some studies have looked for but found no evidence for an endogenous control mechanism-e.g., mosquito (Brammer and Clarin, 1976; Brammer et al., 1978). The report of Hafner and Cook (1980), that cutting the optic nerve in crayfish reduces the multivesicular bodies associated with membrane shedding, is the only other work of which we are aware that suggests a specific mechanism for endogenous control of membrane shedding.

Control mechanisms for membrane shedding: vertebrates. The apparent diversity of control mechanisms for membrane shedding in invertebrates is also found among vertebrate species. As in Limulus, light is required to initiate rod shedding in frogs (Basinger et al., 1976), but the trigger signal appears to spread laterally across the retina so that illumination of only a few rods induces shedding in all of them (Basinger and Gordon, 1982). Numerous experiments indicate that in frogs, control of light-initiated rod shedding resides within the eye itself (e.g.,
Hollyfield and Basinger, 1978; Basinger and Gordon, 1982). When the isolated eyecup is maintained in vitro, light stimulation following a sufficient period of darkness will initiate rod shedding (Besharse et al., 1980; Heath and Basinger, 1983).

In rats the shedding event must be preceded by a period of darkness (Goldman et al., 1980), but light is not required as a trigger and shedding occurs at the appropriate time even in continuous darkness (LaVail, 1976, 1980). A retinal oscillator appears to exert endogenous control of rod shedding, and neither cutting the optic nerves (Teirstein et al., 1980) nor ablating numerous structures in the central nervous system (LaVail and Ward, 1978) abolishes the circadian rhythm of rod shedding in rats. Teirstein et al. (1980) also found, however, that cutting the optic nerve made it impossible to entrain the shedding rhythm to a new lighting cycle. They suggested that rod shedding in rats is controlled by an oscillator in the central nervous system which neurally sets the phase of a retinal oscillator that triggers shedding. The superposition of central and retinal controls in rat may have parallels in Limulus. The 
Limulus brain controls the large daily burst of photoreceptor membrane shedding (Fig. 12). Removal of the brain's efferent input to the retina reveals a persistent complex cycle of membrane metabolism in the retina that apparently does not depend on central control (fig. 13C).

Limulus as a model system. The Limulus visual system is an appropriate model for studying photoreceptor membrane shedding because the process is a robust daily phenomenon controlled by both environmental lighting and the brain. Also, these control mechanisms share characteristics with those found in vertebrates and other invertebrates. Progress is being made in understanding the functional organization of the efferent pathways that mediate central control in Limulus. Efferent fibers from the brain innervate the photoreceptors of all visual organs in Limulus (Fahrenbach, 1975; Battelle et al., 1982; Calman and Chamberlain, 1982; Evans et al., 1983). The efferent fibers transmit synchronous nerve signals from a circadian clock in the brain to the lateral eyes, median ocelli, and ventral photoreceptors on both sides of the animal (Eisele et al., 1982). Furthermore, the efferent fibers allow one eye to influence the sensitivity of another via central neural connections (Barlow et al., 1980a, 1984). Regarding the transmission of efferent optic nerve activity to retinal cells, it has been shown recently that octopamine is synthesized and released by the efferent terminals in the lateral and ventral eyes (Battelle et al., 1982; Evans et al., 1983) and that octopamine mimics many of the physiological effects of efferent activity in the lateral eye (Kass and Barlow, 1981, 1982, 1984). Finally, preliminary studies suggest that efferent fibers to the lateral eye may also contain a neuropeptide (Engbretson and Chamberlain, 1981), although whether it is substance $P$ that is co-localized with octopamine remains unresolved (Engbretson and Chamberlain, 1981; Mancillas et al., 1981; Chamberlain and Engbretson, 1982; Mancillas and Brown, 1984).

\section{References}

Afzelius, B. (1959) Electron microscopy of the sperm tail. Results obtained with a new fixative. J. Biophys. Biochem. Cytol. 5: 269278.

Barlow, R. B., Jr. (1983) Circadian rhythms in the Limulus visual system. J. Neurosci. 3: 856-870.

Barlow, R. B., Jr., and S. C. Chamberlain (1980) Light and a circadian clock modulate structure and function in Limulus photoreceptors. In The Effects of Constant Light on Visual Processes T. P. Williams and B. N. Baker, eds. pp. 247-269, Plenum Press, New York.

Barlow, R. B., Jr., S. J. Bolanowski, Jr. and M. L. Brachman (1977) Efferent optic nerve fibers mediate circadian rhythms in the Limulus eye. Science 197: 86-89.

Barlow, R. B., Jr., S. C. Chamberlain, S. J. Bolanowski, Jr., L. A. Galway, Jr., and D. P. Joseph (1980a) One eye can increase the sensitivity of another in Limulus. Soc. Neurosci. Abstr. 6: 344.

Barlow, R. B., Jr., S. C. Chamberlain, and J. Z. Levinson (1980b) Limulus brain modulates structure and function of the lateral eyes. Science 210: 1037-1039.

Barlow, R. B., Jr., S. C. Chamberlain, and L. A. Westerman (1984) Circadian clock in the brain mediates ncural interactions among the eyes of Limulus. Science, submitted.

Basinger, S. F., and W. C. Gordon (1982) Local stimulation induces shedding throughout the frog retina. Vision Res. 22: 1533-1538.

Basinger, S. F., R. Hoffman, and M. Matthes (1976) Photoreceptor shedding is initiated by light in the frog retina. Science 194: 10741076 .

Batra, R., and R. B. Barlow, Jr. (1982) Efferent control of pattern vision in Limulus. Soc. Neurosci. Abstr. 8: 49.

Battelle, B. -A., J. A. Evans, and S. C. Chamberlain (1982) Efferent fibers to Limulus eyes synthesize and release octopamine. Science 216: $1250-1252$.

Behrens, M. E. (1974) Photomechanical changes in the ommatidia of the Limulus lateral eye during light and dark adaptation. J. Comp. Physiol. 89: 45-57.

Behrens, M. E., and W. Krebs (1976) The effects of light-dark adap- tation on the ultrastructure of Limulus lateral eye retinular cells. J. Comp. Physiol. 107: 77-96.

Besharse, J. C., R. O. Terrill, and D. A. Dunis (1980) Light-evoked disc shedding in vitro: Relationship to medium bicarbonate concentration. Invest. Ophthalmol. Vis. Sci. 19: 1512-1517.

Blest, A. D. (1978) The rapid synthesis and destruction of photoreceptor membrane by a dinopid spider: A daily cycle. Proc. R. Soc. Lond. Biol. 200: 463-483.

Blest, A. D., L. Kao, and K. Powell (1978) Photoreceptor membrane breakdown in the spider Dinopis: The fate of rhabdomere products. Cell Tissue Res. 195: 425-444.

Blest, A. D., S. Stowe, W. Eddey, and D. S. Williams (1982) The local deletion of a microvillar cytoskeleton from photoreceptors of tipulid flies during membrane turnover. Proc. R. Soc. Lond. Biol. 215: 469 479.

Brammer, J. D., and B. Clarin (1976) Changes in volume of the rhabdom in the compound eye of Aedes aegypti L. J. Exp. Zool. 195: 33-40.

Brammer, J. D., P. J. Stein, and R. A. Anderson (1978) Effect of light and dark adaptation upon the rhabdom in the compound eye of the mosquito. J. Exp. Zool. 206: 151-156.

Calman, B. G., and S. C. Chamberlain (1982) Distinct lobes of Limulus ventral photoreceptors. II. Structure and ultrastructure. J. Gen. Physiol. 80: 839-862.

Chamberlain, S. C., and R. B. Barlow, Jr. (1977) Morphological correlates of efferent circadian activity and light adaptation in the Limulus lateral eye. Biol. Bull. 153: 418-419.

Chamberlain, S. C., and R. B. Barlow, Jr. (1979) Light and efferent activity control rhabdom turnover in Limulus photoreceptors. Science 206: 361-363.

Chamberlain, S. C., and R. B. Barlow, Jr. (1981) Modulation of retinal structure in Limulus lateral eye: Interactions of light and efferent inputs. Invest. Ophthalmol. Vis. Sci. 20: 75 .

Chamberlain, S. C., and G. A. Engbretson (1982) Neuropeptide immunoreactivity in Limulus. I. Substance P-like immunoreactivity in the lateral eye and protocerebrum. J. Comp. Neurol. 208: 304-315.

Cohen, H. A. (1973) Quantitative aspects of the eccentric cell dendrite of the lateral eye of Limulus. J. Neurocytol. 2: 429-439.

Eguchi, E., and T. H. Waterman (1967) Changes in retinal fine structure induced in the crab Libinia by light and dark adaptation. $\mathrm{Z}$. Zellforsch. 79: 209-229.

Eguchi, E., and T. H. Waterman (1976) Freeze-etch and histochemical evidence for cycling in crayfish photoreceptor membranes. Cell Tissue Res. 169: 419-434.

Eisele, L. E., L. Kass, and R. B. Barlow, Jr. (1982) Circadian clock generates optic nerve activity in the excised Limulus brain. Biol. Bull. 163: 382 .

Engbretson, G. A., and S. C. Chamberlain (1981) Efferent fibers to the Limulus lateral eye may contain the neuropeptide ranatensin. Invest. Ophthalmol. Vis. Sci. Suppl. 20: 75.

Evans, J. A., S. C. Chamberlain, and B. -A. Battelle (1983) Autoradiographic localization of newly synthesized octopamine to retinal efferents in the Limulus visual system. J. Comp. Neurol. 219: 369383.

Fahrenbach, W. H. (1968) The morphology of the eyes of Limulus. I. Cornea and epidermis of the compound eye. Z. Zellforsch. 87: 278291.

Fahrenbach, W. H. (1969) The morphology of the eyes of Limulus. II. Ommatidia of the compound eye. Z. Zellforsch. 93: 451-483.

Fahrenbach, W. H. (1973) The morphology of the Limulus visual system. V. Protocerebral neurosecretion and ocular innervation. $Z$. Zellforsch. 144: 153-166.

Fahrenbach, W. H. (1975) The visual system of the horseshoe crab Limulus polyphemus. Int. Rev. Cytol. 41: 285-349.

Fahrenbach, W. H. (1981) The morphology of the horseshoe crab (Limulus polyphemus) visual system. VII. Innervation of photoreceptor neurons by neurosecretory efferents. Cell Tissue Res. 216: 655659.

Fain, G. L., and J. E. Lisman (1981) Membrane conductances of photoreceptors. Prog. Biophys. Mol. Biol. 37: 91-147.

Fein, A., and E. Z. Szuts (1982) Photoreceptors: Their Role in Vision, Cambridge University Press, New York.

Goldman, A I., P. S. Teirstein, and P. J. O'Brien (1980) The role of ambient lighting in circadian disc shedding in the rod outer segment of the rat retina. Invest. Ophthalmol. Vis. Sci. 19: 1257-1267.

Hafner, G. S., and M. D. Cook (1980) Light induced rhabdom degra- 
dation in the crayfish in vivo and in vitro. Invest. Ophthalmol. Vis Sci. Suppl. 19: 252.

Hafner, G. S., G. Hammond-Soltis, and T. Tokarski (1980) Diurnal changes of lysosome-related bodies in the crayfish photoreceptor cells. Cell Tissue Res. 206: 319-332.

Heath, A. R. and S. F. Basinger (1983) Frog rod segment shedding in vitro: Histologic and electrophysiologic observations. Invest. Ophthalmol. Vis. Sci. 24: 277-284.

Hollyfield, J. G., and S. F. Basinger (1978) Photoreceptor shedding can be initiated within the eye. Nature 274: 794-796.

Horridge, G. A., J. Duniec, and L. Marcelja (1981) A 24-hour cycle in single locust and mantis photoreceptors. J. Exp. Biol. 91: 307-322.

Itaya, S. K. (1976a) Rhabdom changes in the shrimp, Palaemonetes. Cell Tissue Res. 166: 265-273.

Itaya, S. K. (1976b) Light and dark adaptational changes in the accessory eye of the shrimp, Palaemonetes. Tissue Cell 8: 583-590.

Kaplan, E., and R. B. Barlow, Jr. (1980) Circadian clock in Limulus brain increases response and decreases noise of retinal photoreceptors. Nature 286: 393-395.

Kass, L., and R. B. Barlow, Jr. (1981) Octopamine increases the ERG of the Limulus lateral eye. Biol. Bull. 159: 487.

Kass, L., and R. B. Barlow, Jr. (1982) Efferent neurotransmission of circadian rhythms in Limulus lateral eye: Single cell studies. Biol. Bull. 163: 386.

Kass, L., and R. B. Barlow, Jr. (1984) Efferent neurotransmission of circadian rhythms in the Limulus lateral eye. I. Octopamine-induced increases in retinal sensitivity. J. Neurosci. 1: 908-917.

Kataoka, S., and T. Y. Yamamoto (1983) Fine structure and formation of the photoreceptor in Octopus ocellatus. Biol. Cell 49: 45-54.

Kataoka, S., T. Y. Yamamoto, A. Tonosaki, and H. Washioka (1982) Ultrastructural study of photoreceptor membrane turnover in an invertebrate retina. In The Structure of the Eye, J. G. Hollyfield, ed., pp. 35-44, Elsevier Biomedical Press, New York.

Krebs, W., and B. Schaten (1976) The lateral photoreceptor of the barnacle, Balanus eburneus. Quantitative morphology and fine structure. Cell Tissue Res. 168: 193-207.

Lasansky, A. (1967) Cell junctions in ommatidia of Limulus. J. Cell Biol. 33: 365-384.

LaVail, M. M. (1976) Rod outer segment disc shedding in the rat retina: Relationship to cyclic lighting. Science 194: 1071-1073.

LaVail, M. M. (1980) Circadian nature of rod outer segment disc shedding in the rat. Invest. Ophthalmol. Vis. Sci. 19: 407-411.

LaVail, M. M., and P. A. Ward (1978) Studies on the hormonal control of circadian outer segment disc shedding in the rat retina. Invest. Ophthalmol. Vis. Sci. 17: 1189-1193.
Mancillas, J. R., and M. R. Brown (1984) Neuropeptide modulation of photosensitivity. I. Presence, distribution, and characterization of a substance P-like peptide in the lateral eye of Limulus. J. Neurosci. 4: 832-846.

Mancillas, J. R., J. F. McGinty, and A. I. Selverston (1981) Distribution of enkephalin, substance $\mathrm{P}$ and serotonin in the visual system of the lobster, crayfish and horseshoe crab. Soc. Neurosci. Abstr. 7: 223.

Miller, W. H. (1957) Morphology of the ommatidia of the compound eye of Limulus. J. Biophys. Biochem. Cytol. 3: 421-427.

Miller, W. H. (1958) Fine structure of some invertebrate photoreceptors. Ann. N. Y. Acad. Sci. 74: 204-209.

Miller, W. H., and D. F. Cawthon (1974) Pigment granule movement in Limulus photoreceptors. Invest. Ophthalmol. 13: 401-405.

Nässel, D. R., and T. H. Waterman (1979) Massive diurnally modulated photoreceptor membrane turnover in crab light and dark adaptation. J. Comp. Physiol. 131: 205-216.

Ratliff, F. (1974) Studies on Excitation and Inhibition in the Retina, Rockefeller University Press, New York.

Stowe, S. (1980) Rapid synthesis of photoreceptor membrane and assembly of new microvilli in a crab at dusk. Cell Tissue Res. 211. $419-440$.

Teirstein, P. S., A. I. Goldman, and P. J. O’Brien (1980) Evidence for both local and central regulation of rat rod outer segment disc shedding. Invest. Ophthalmol. Vis. Sci. 19: 1268-1273.

Trump. B. F., E. A. Smuckler, and E. P. Benditt (1961) A method for staining epoxy sections for light microscopy. J. Ultrastruct. Res. 5: 343.

White, R. H. (1967) The effect of light and light deprivation upon the ultrastructure of the larval mosquito eye. II. The rhabdom. J. Exp. Zool. 166: 405-426.

White, R. H. (1968) The effect of light and light deprivation upon the ultrastructure of the larval mosquito eye. III. Multivesicular bodies and protein uptake. J. Exp. Zool. 169: 261-278.

White, R. H., and N. A. Michaud (1981) Disruption of insect photoreceptor membrane by divalent ions: Dissimilar sensitivity of lightand dark-adapted mosquito rhabdomeres. Cell Tissue Res. 216: 403411.

Williams. D. S. (1980) $\mathrm{Ca}^{++}$-induced structural changes in photoreceptor microvilli of diptera. Cell Tissue Res. 206: 225-232.

Williams, D. S. (1982a) Rhabdom size and photoreceptor membrane turnover in a muscoid fly. Cell Tissue Res. 226: 629-639.

Williams, D. S. (1982b) Ommatidial structure in relation to turnover of photoreceptor membrane in the locust. Cell Tissue Res. 225: 595617. 